\title{
A primal-dual active set method and predictor-corrector mesh adaptivity for computing fracture propagation using a phase-field approach
}

\author{
Timo Heister ${ }^{\mathrm{a}}$, Mary F. Wheeler ${ }^{\mathrm{b}}$, Thomas Wick Th, $, 1, *^{\mathrm{b}}$ \\ ${ }^{a}$ Mathematical Sciences, Clemson University \\ Clemson, SC 29634, USA \\ ${ }^{b}$ The Institute for Computational Engineering and Sciences, \\ The University of Texas at Austin, \\ Austin, Texas 78712, USA \\ ${ }^{c}$ Johann Radon Institute for Computational and Applied Mathematics, \\ Austrian Academy of Sciences, \\ 4040 Linz, Austria
}

\begin{abstract}
In this paper, we consider phase-field-based fracture propagation in elastic media. The main purpose is the development of a robust and efficient numerical scheme. To enforce crack irreversibility as a constraint, we use a primal-dual active set strategy, which can be identified as a semi-smooth Newton's method. The active set iteration is merged with the Newton iteration for solving the fully-coupled nonlinear partial differential equation discretized using finite elements, resulting in a single, rapidly converging nonlinear scheme. It is well known that phase-field models require fine meshes to accurately capture the propagation dynamics of the crack. Because traditional estimators based on adaptive mesh refinement schemes are not appropriate, we develop a predictorcorrector scheme for local mesh adaptivity to reduce the computational cost. This method is both robust and efficient and allows us to treat temporal and spatial refinements and to study the influence of model regularization parameters. Finally, our proposed approach is substantiated with different numerical tests for crack propagation in elastic media and pressurized fracture propagation in homogeneous and heterogeneous media.
\end{abstract}

Keywords: phase-field, fracture mechanics, predictor corrector mesh adaptivity, primal-dual active set 2010 MSC: 65K15, 65M60, 65N30, 74F99

\footnotetext{
* Corresponding author

Email addresses: heister@clemson.edu (Timo Heister), mfw@ices.utexas.edu (Mary F. Wheeler), twick@ices.utexas.edu (Thomas Wick)

${ }^{1} \mathrm{~T}$. Wick is currently employed by RICAM, an institute by the Austrian Academy of Sciences. He is still linked to ICES through a JT Oden Faculty Research Visitor Fellowship; a support that is gratefully acknowledged.
}

Preprint submitted to Elsevier

January 6, 2015

(C) 2015. This manuscript version is made available under the Elsevier user license http://www.elsevier.com/open-access/userlicense/1.0/ 


\section{Introduction}

Presently, crack propagation in elastic and porous media is one of the major research topics in mechanical, energy, and environmental engineering. In recent years, variational approaches and phase-field techniques for brittle fracture have gained increased interest from studies presented in $[1,2,3,4,5,6,7,8]$. Instead of modeling the discontinuities explicitly (like in the extended finite element method [9] or generalized finite elements [10]), the lower-dimensional crack surface is approximated by a phase-field function. This is an indicator function, which introduces a diffusive transition zone (brittle or mushy-zone are also common expressions depending on the discipline) between the broken and the unbroken material. Most importantly, fracture nucleation, propagation, kinking, and curvilinear paths are automatically included in the model; postprocessing of stress intensity factors and remeshing resolving the crack path are avoided.

From a numerical viewpoint, the key aspects of the phase-field-based fracture propagation approach are techniques that include fine meshes around the crack, the solution of the resulting energy functional or its characterizing weak formulated Euler-Lagrange equations, and the enforcement of the irreversibility of crack growth. To solve for the admissible displacements and the phase-field values, we use the Euler-Lagrange equations to employ a Galerkin finite element scheme. This means that we obtain a critical point rather than a global minimizer. Here, a non-symmetric semi-linear form is obtained, which can not be characterized as a minimization procedure [11]. However, such a formulation allows the extension to quasi-static poroelasticity $[12,13]$. In addition, the Euler-Lagrange form allows more complicated interface laws in coupling fracture propagation with other physics; a motivation can be found in [14].

For the crack irreversibility, we employ a robust primal-dual active set strategy based on [15] (as often used for contact problems, e.g. [16]). An active set method is highly attractive because it can be interpreted as a semi-smooth Newton method and allows for fast (super-linear) convergence. In contrast to other methods such as simple penalization or augmented Lagrangian methods, the adjustment of additional parameters is not necessary. For an overview of algorithms we refer the reader to [17].

Within the active set iteration, the coupled Euler-Lagrange PDE system is addressed monolithically in which both equations, elasticity and phase-field, are solved simultaneously. We propose a robust numerical scheme to treat the non-convexity of the regularized energy functional and resulting consequences on the Euler-Lagrange system.

Phase-field methods require fine meshes in crack regions resulting in high computational cost. To address this issue, we propose a predictor-corrector local mesh adaptivity scheme. It does not require any a priori knowledge of the crack path and therefore, the number of degrees of freedom grows during the computation. Therefore, it is much more efficient than global as well as a priori 
local mesh refinement; because there, the final numbers of degrees of freedom are already present in the initial set-up.

We briefly summarize the novelty of results presented in this paper:

- a well-known, robust, semi-smooth Newton method for treating variational inequalities is combined with phase-field for fracture propagation;

- a robust (heuristic) procedure based on extrapolation of known phasefield values to cope with the non-convexity of the energy functional allows formulation of a monolithic scheme for the forward problem;

- an efficient predictor-corrector mesh adaptivity scheme is developed that allows the mesh to grow during the computation rather than a priori refinement. The algorithm guarantees that the local mesh size $h$ is always smaller than the constant phase-field regularization parameter $\varepsilon$;

- insensitivity of crack growth with respect to the mesh structure; a crucial property of the algorithm.

The paper is organized as follows: In Section 2, we introduce the notation and recapitulate the energy functional. Then, in Section 3, the primaldual active set strategy to enforce the irreversibility condition on the crack growth is derived. The semi-smooth Newton method for the active set is then merged with the Newton method for the nonlinear PDE, which is solved in terms of monolithically-coupled Euler-Lagrange equations. Section 4 introduces a predictor-corrector local adaptive mesh refinement technique. Finally, in Section 5 several numerical tests and benchmarks are used to substantiate our algorithmic developments. These tests include fracture propagation in elasticity and pressurized cracks in heterogeneous elasticity as well as sensitivities studies with respect to the mesh.

\section{Notation and Equations}

In this section, we provide the basic notation and the weak form of the underlying equations. We denote the $L^{2}$ scalar product with $(\cdot, \cdot)$ as frequently used in the literature. In the following, let $\Omega \subset \mathbb{R}^{2}$, be a smooth, open, connected, and bounded set. We assume that the crack $\mathcal{C}$ is a 1-dimensional set, not necessarily connected, that is contained in $\Omega$. We assume (possibly timedependent non-homogeneous) Dirichlet boundaries conditions on (part of) the boundary $\partial \Omega_{D} \subseteq \partial \Omega$.

\subsection{The energy functional}

The Francfort-Marigo functional [1] (based on the Mumford-Shah functional) describes the energy of a crack in an elastic medium as

$$
E(\boldsymbol{u}, \mathcal{C})=\frac{1}{2}(\sigma, e(\boldsymbol{u}))-\int_{\mathcal{C}} \tau \cdot \boldsymbol{u} d s+G_{c} H^{1}(\mathcal{C}),
$$


where $\boldsymbol{u}: \Omega \rightarrow \mathbb{R}^{2}$ is the displacement, $\sigma=\sigma(\boldsymbol{u})$ the stress tensor, and $e(\boldsymbol{u})$ the symmetric strain tensor defined as

$$
e(\boldsymbol{u}):=\frac{1}{2}\left(\nabla \boldsymbol{u}+\nabla \boldsymbol{u}^{T}\right) .
$$

The Hausdorff measure $H^{1}(\mathcal{C})$ denotes the length of the crack and is multiplied by the energy release rate $G_{c}>0$. Traction forces on the crack boundary are denoted by $\tau$.

In $[12,13]$ the functional $(1)$ was augmented with a pressure term in order to account for pressurized fracture propagation. The idea is to assume $-p I$ as a leading order of stress in $\mathcal{C}$ and standard calculations yield

$$
\int_{\mathcal{C}} \tau \cdot \boldsymbol{u} d s=\int_{\mathcal{C}} \sigma n \cdot \boldsymbol{u} d s=-\int_{\mathcal{C}} p n \cdot \boldsymbol{u} d s=\int_{\Omega} p \nabla \cdot \boldsymbol{u}+\int_{\Omega} \nabla p \boldsymbol{u} .
$$

Combining this term with the poroelastic stress $\sigma_{\text {poro }}=\sigma-\alpha p I$ (with Biot's coefficient $\alpha \in[0,1]$ ), and including the phase-field variable $\varphi$ (see $[12,13]$ ), we obtain the representation as included in the following functional:

$$
E(\boldsymbol{u}, \mathcal{C})=\frac{1}{2}(\sigma, e(\boldsymbol{u}))-(\alpha-1)\left(\varphi^{2} p, \operatorname{div} \boldsymbol{u}\right)+\left(\varphi^{2} \nabla p, \boldsymbol{u}\right)+G_{c} H^{1}(\mathcal{C})
$$

where $p: \Omega \rightarrow \mathbb{R}$ is a given pressure.

Remark 2.1. In poroelastic applications, the pressure $p$ is obtained from solving a pressure Darcy equation. This coupled system is solved with a fixed-stress iteration $[18,19]$ in which the pressure equation and elasticity equation are solved sequentially. The pressure gradient term $\varphi^{2} \nabla p$ is obtained from the interface where the fluid pressure in the crack interacts with poroelastic fluid flow $[12,14]$. For a given, piece-wise constant, pressure this term is zero and neglected in the rest of this paper. In addition, Biot's coefficient is chosen as $\alpha=0$. Both terms appear in situations as investigated in [20,21, 22].

Remark 2.2. One easily observes that the pressurized crack phase-field model contains crack propagation in pure elasticity as special case if $p=0$. Consequently, we can model and compute various fracture propagation scenarios within elasticity employing our approach, which is demonstrated in the numerical examples.

In the remainder of this paper, we represent the crack $\mathcal{C}$ using a continuous phase-field variable $\varphi: \Omega \rightarrow[0,1]$, where a value of 0 represents the crack region. This introduces a diffusive transition zone between the broken and unbroken material controlled by the regularization parameter $\varepsilon>0$.

Remark 2.3. Relating $\Gamma$ convergence and spatial discretization, it is well-known that $h=o(\varepsilon)$. In this work, studies for investigating $\varepsilon$ and its relationship to the mesh size parameter $h$ are carried out. In this respect, we notice that the $\varepsilon$ might be considered as a (fixed) length-scale parameter that might have a physical meaning, which is subject of investigation in gradient-type material modeling $([23,4]$ and references cited therein). 
Using $\varepsilon$ allows us to replace the crack surface energy $G_{c} H^{1}(\mathcal{C})$ in $(1)$ by an elliptic Ambrosio-Tortorelli functional $[24,25] G_{c}\left(\frac{1}{2 \varepsilon}\|1-\varphi\|^{2}+\frac{\varepsilon}{2}\|\nabla \varphi\|^{2}\right)$ and formulating a global constitutive dissipation functional for a rate independent fracture process. In addition, we allow for correct modeling of shear forces under compression, following [4] we decompose the stress tensor (i.e., the energy) into tensile $\sigma^{+}$and compressive $\sigma^{-}$parts, which then yields:

$$
\begin{aligned}
E_{\varepsilon}(\boldsymbol{u}, \varphi)= & \frac{1}{2}\left(\left((1-\kappa) \varphi^{2}+\kappa\right) \sigma^{+}(\boldsymbol{u}), e(\boldsymbol{u})\right)+\frac{1}{2}\left(\sigma^{-}(\boldsymbol{u}), e(\boldsymbol{u})\right) \\
& -(\alpha-1)\left(\varphi^{2} p, \operatorname{div} \boldsymbol{u}\right) \\
& +G_{c}\left(\frac{1}{2 \varepsilon}\|1-\varphi\|^{2}+\frac{\varepsilon}{2}\|\nabla \varphi\|^{2}\right)
\end{aligned}
$$

where $\kappa$ is a positive regularization parameter for the elastic energy, with $\kappa \ll \varepsilon$ and standard linear elasticity with the stress-strain relationship

$$
\sigma:=\sigma(\boldsymbol{u})=2 \mu e(\boldsymbol{u})+\lambda \operatorname{tr}(e(\boldsymbol{u})) I .
$$

Remark 2.4 (The role of the regularization parameter $\kappa$ ). The parameter $\kappa$ regularizes the bulk energy. It is evident that this parameter should be as small as possible to avoid over-estimation of bulk energy (resulting in an underestimation of the surface crack energy). In practice this means: the larger the value of $\kappa$, the slower the crack will grow. There are theoretical studies in which this parameter is not used at all [26] and if it is, it should be chosen as $\kappa=o(\varepsilon)$ such that $\Gamma$ convergence results remain valid. In [2], the authors provide explanations of its role. In [6], the authors state that in several of their numerical studies they observed that $\kappa$ could be set to zero. Instead of setting $\kappa$ to zero, we chose small values in the order of $10^{-10}$ and found that this choice does not influence the solutions.

Furthermore, in the energy functional (3), we use (see $[5,6]$ )

$$
\begin{aligned}
\sigma^{+} & =2 \mu e^{+}+\lambda<\operatorname{tr}(e)>I, \\
\sigma^{-} & =2 \mu\left(e-e^{+}\right)+\lambda(\operatorname{tr}(e)-<\operatorname{tr}(e)>) I,
\end{aligned}
$$

and

$$
e^{+}=P \Lambda^{+} P^{T},
$$

where $\langle\cdot\rangle$ is the positive part of a function. Moreover, for $d=2$,

$$
\Lambda^{+}:=\Lambda^{+}(\boldsymbol{u}):=\left(\begin{array}{cc}
<\lambda_{1}(\boldsymbol{u})> & 0 \\
0 & <\lambda_{2}(\boldsymbol{u})>
\end{array}\right) .
$$

where $\lambda_{1}(\boldsymbol{u})$ and $\lambda_{2}(\boldsymbol{u})$ are the eigenvalues of the strain tensor $e$, and $v_{1}(\boldsymbol{u})$ and $v_{2}(\boldsymbol{u})$ the corresponding (normalized) eigenvectors. Finally, the matrix $P$ 
is defined as $P:=P(\boldsymbol{u}):=\left(v_{1}, v_{2}\right)$; namely, it consists of the column vectors $v_{i}, i=1,2$.

To compute the crack path, we seek vector-valued displacements $\boldsymbol{u}$ and the scalar-valued phase-field $\varphi$ such that the total energy $E_{\varepsilon}(\boldsymbol{u}, \varphi)$ is minimized. This problem is completed with an inequality constraint with the condition that cracks keep their length or grow in time:

Formulation 1 (Fracture energy minimization with irreversibility constraint). Find $\boldsymbol{u}$ and $\varphi$ for almost all times $t$ such that

$$
\min E_{\varepsilon}(\boldsymbol{u}, \varphi) \quad \text { s.t. } \partial_{t} \varphi \leq 0 .
$$

The time $t$ enters through time-dependent boundary conditions, e.g., $\boldsymbol{u}=\boldsymbol{u}(t)=$ $\boldsymbol{g}(t)$ on $\partial \Omega_{D}$ with a prescribed boundary function $\boldsymbol{g}(t)$ of Dirichlet-type (see Section 5.4) or through time-dependent right hand side forces, e.g., $p:=p(t)$ as used in Section 5.6.

Remark 2.5. The fracture energy minimization is formulated in terms of quasistatic assumptions. Here, time $t$ refers rather to a loading step parameter than a true time.

\subsection{The Euler-Lagrange equations}

Finally, the weak form is derived from problem formulation (4) by taking the first variation with respect to the solution variables. For this, let $V:=H_{0}^{1}(\Omega)$, $W_{i n}:=\left\{w \in H^{1}(\Omega) \mid w \leq \varphi^{n-1} \leq 1\right.$ a.e. on $\left.\Omega\right\}$ (where $\varphi^{n-1}$ denotes the previous time step solution of $\varphi$ ), and $W:=H^{1}(\Omega)$.

Formulation 2 (Euler-Lagrange system of Formulation 1). Find $(\boldsymbol{u}, \varphi) \in V \times$ $W$ with

$$
\begin{gathered}
\left(\left((1-\kappa) \varphi^{2}+\kappa\right) \sigma^{+}(\boldsymbol{u}), e(\boldsymbol{w})\right)+\left(\sigma^{-}(\boldsymbol{u}), e(\boldsymbol{w})\right) \\
-(\alpha-1)\left(\varphi^{2} p, \operatorname{div} \boldsymbol{w}\right)=0 \quad \forall \boldsymbol{w} \in V
\end{gathered}
$$

as well as

$$
\begin{aligned}
& (1-\kappa)\left(\varphi \sigma^{+}(\boldsymbol{u}): e(\boldsymbol{u}), \psi-\varphi\right)-2(\alpha-1)(\varphi p \operatorname{div} \boldsymbol{u}, \psi-\varphi) \\
& +G_{c}\left(-\frac{1}{\varepsilon}(1-\varphi, \psi-\varphi)+\varepsilon(\nabla \varphi, \nabla \psi-\varphi)\right) \geq 0 \quad \forall \psi \in W_{i n} \cap L^{\infty}(\Omega) .
\end{aligned}
$$




\section{Solution Algorithm and Discretization}

This first key section introduces the solution algorithm. In Sections 3.1 to 3.4, we first formulate a primal-dual active set strategy as the overall solution algorithm; including continuous forms and their corresponding discretization. Specifically, this penalization strategy is required to treat the constraint $\partial_{t} \varphi \leq 0$ for crack irreversibility. We recall that the system is quasi-static and time enters only through this constraint. In the second part that comprises the Sections 3.5 and 3.6, we focus on the PDE that is required to be solved in each Newton active set step. This PDE is represented in terms of the monolithically-coupled Euler-Lagrange equations. In particular, we explain a technique to convexify the energy functional in order to obtain a positive definite Hessian matrix. Finally, we briefly explain the spatial discretization and the block structure of the linear equation system.

\subsection{Setup}

We emphasize the minimization problem (see Formulation (1))

$$
\begin{aligned}
& \min E_{\varepsilon}(\boldsymbol{u}, \varphi) \\
& \text { subject to } \partial_{t} \varphi \leq 0 .
\end{aligned}
$$

is quite unusual since the forward problem is quasi-static without any explicit time derivatives and the time-dependence appears only in the inequality constraint. For the following, we set $U=(\boldsymbol{u}, \varphi) \in V \times W$. Discretizing

$$
\partial_{t} \varphi \approx \frac{\varphi\left(t^{n+1}\right)-\varphi\left(t^{n}\right)}{\delta t},
$$

with time step size $\delta t:=t^{n+1}-t^{n}$, the problem can be rewritten as

$$
\begin{aligned}
& \min E_{\varepsilon}(U) \\
& \text { subject to } U \leq \bar{U} \text { on } \Phi,
\end{aligned}
$$

where $\Phi=0 \times W$, so that the constraint acts on the phase-field variable only, and $\bar{U}$ is the solution from the last time-step (or the initial condition).

\subsection{Newton iteration}

We now briefly describe Newton's method for solving the unconstrained minimization problem $\min E_{\varepsilon}(U)$ in (7). We construct a sequence $U^{0}, U^{1}, \ldots, U^{N}$ with

$$
U^{k+1}=U^{k}+\delta U^{k},
$$

where the update $\delta U^{k}$ is computed as the solution of the linear system (details in Section 3.5):

$$
\nabla^{2} E_{\varepsilon}\left(U^{k}\right) \delta U^{k}=-\nabla E_{\varepsilon}\left(U^{k}\right) .
$$


If we assume the constraints on the phase-field (8) hold for the initial guess $U^{0}$ (we will start with the solution from the last time step, which satisfies the constraint), the condition

$$
\delta U^{k} \leq 0 \text { on } \Phi,
$$

implies $U^{k+1}=U^{k}+\delta U^{k} \leq U^{k} \leq \cdots \leq U^{0} \leq \bar{U}$ on $\Phi$.

\subsection{A primal-dual active set strategy for crack irreversibly}

Let us now derive a primal-dual active set strategy for the linear system (9) with constraint (10) to be solved in each Newton step:

$$
\begin{array}{r}
\nabla^{2} E_{\varepsilon}\left(U^{k}\right) \delta U^{k}=-\nabla E_{\varepsilon}\left(U^{k}\right), \\
\text { with } \delta U^{k} \leq 0 \text { on } \Phi .
\end{array}
$$

Here, in contrast to the work originally proposed in [15], the constraint relates two time states (within a time-stepping scheme) rather than spatial constraints.

In the following, we shorten the notation by dropping the index $k$ of Newton's method and setting $G:=\nabla^{2} E_{\varepsilon}\left(U^{k}\right), F:=-\nabla E_{\varepsilon}\left(U^{k}\right)$ (this also highlights that our operator $G$ is fixed in this section), and $\delta U:=\delta U^{k}$. We note that ideally $G$ is symmetric positive definite in order to employ a robust solution scheme. The previous system can be written as a minimization problem

$$
\begin{aligned}
& \min \frac{1}{2}(\delta U, G \delta U)-(F, \delta U), \\
& \text { with } \delta U \leq 0 \text { on } \Phi .
\end{aligned}
$$

Following [15] the minimization problem (11) can be solved using a primal-dual active set strategy, which can also be viewed as a semi-smooth Newton method. We briefly recapitulate the most important steps since their understanding is crucial for our final algorithm. Using a Lagrange multiplier $\lambda \in 0 \times W^{*}$ (where $W^{*}$ is the dual space of $W$ ), the minimization problem with constraint can be written as a system of equations:

$$
\begin{aligned}
(G \delta U, Z)+(\lambda, Z) & =(F, Z) \forall Z \in V \times W, \\
C(\delta U, \lambda) & =0
\end{aligned}
$$

where

$$
C(\delta U, \lambda)=\lambda-\max (0, \lambda+c \delta U),
$$

for a given $c>0$. The max operation is understood in the point-wise sense. Since we require the Lagrange multiplier only for the phase-field variable $\varphi$, we can assume zero displacements (alternatively, one needs to restrict $\delta U$ to the phase-field in the definition of $C$ ).

The primal-dual active set strategy replaces the condition $C(\delta U, \lambda)=0$ by $\delta U=0$ on the to be determined active set $\mathcal{A}$ and $\lambda=0$ on the inactive set $\mathcal{I}$. In other words, the active set is the subdomain in which the constraint applies and no PDE is solved. In the inactive set, the PDE is solved while the constraint is satisfied.

The active set algorithm then reads: 
Algorithm 3.1. Repeat for $k=0, \ldots$ until the active set $\mathcal{A}_{k}$ does not longer change:

1. Compute active set:

$$
\begin{aligned}
\mathcal{A}_{k} & =\left\{x \mid \lambda^{k}(x)+c \delta U^{k}(x)>0\right\}, \\
\mathcal{I}_{k} & =\left\{x \mid \lambda^{k}(x)+c \delta U^{k}(x) \leq 0\right\} .
\end{aligned}
$$

2. Find $\delta U^{k+1} \in V \times W$ and $\lambda^{k+1} \in 0 \times W^{*}$

$$
\begin{aligned}
\left(G \delta U^{k+1}, Z\right)+\left(\lambda^{k+1}, Z\right) & =(F, Z) \forall Z \in V \times W, \\
\left(\delta U^{k+1}, \mu\right) & =0 \text { on } \mathcal{A}_{k} \forall \mu \in 0 \times W^{*}, \\
\lambda^{k+1} & =0 \text { on } \mathcal{I}_{k} .
\end{aligned}
$$

So far, the algorithm has been formulated on a continuous level. Now, we employ a finite element discretization by first subdividing the domain into quadrilateral elements. Displacements $\boldsymbol{u}$ and the phase-field variable $\varphi$ are discretized using $H^{1}$-conforming bilinear elements, i.e., the ansatz and test space uses $Q_{1}^{c}$-finite elements. Consequently, the discrete spaces are conforming such that $V_{h} \times W_{h} \subset V \times W$. A discretized version of step 2 then results in a linear system with the following block structure:

$$
\left(\begin{array}{cc}
G & B \\
B^{T} & 0
\end{array}\right)\left(\begin{array}{c}
\delta U_{h}^{k+1} \\
\lambda_{h}^{k+1}
\end{array}\right)=\left(\begin{array}{c}
F \\
0
\end{array}\right)
$$

By using a quadrature only in the support points of $\lambda_{h}^{k}, B^{T}$ becomes diagonal and $\lambda_{h}^{k}$ can be eliminated from the system. The equations $B^{T} \delta U_{h}^{k+1}=0$ will be handled via linear constraints used to eliminate equations in the $G$ block where the phase-field is constrained (on $\mathcal{A}_{k}$ ). The eliminated equations are exactly those where the $i$-th entry of $\lambda_{h}^{k+1}$ is non-zero. Therefore, the linear solve simplifies to

$$
\hat{G} \delta U_{h}^{k+1}=\hat{F},
$$

where $\hat{G}$ and $\hat{F}$ stem from $G$ and $F$ by removing the constrained rows from the system (we opt to restore symmetry by using Gaussian elimination on the columns in our implementation).

Finally, each entry of $\lambda_{h}$ can be computed from $U_{h}^{k+1}$ using

$$
(B)_{i i}\left(\lambda_{h}^{k}\right)_{i}=(F)_{i}-\left(G \delta U_{h}^{k}\right)_{i},
$$

which is needed in the computation of the active set $\mathcal{A}$ in each step. The index $i$ is in the active set $\mathcal{A}_{k}$ if

$$
\left(B^{-1}\right)_{i i}\left(F-G \delta U_{h}^{k}\right)_{i}+c\left(\delta U_{h}^{k}\right)_{i}>0,
$$

and in the inactive set $\mathcal{I}$ otherwise.

Remark 3.1. Note that we require the matrix $G$ and right-hand side $F$ without constraints in (13) and (14). 


\subsection{The final algorithm: Combining two Newton iterations}

In our implementation we combine two Newton methods (active set and the nonlinear iteration for the PDE solution; see Section 3.5) into a single update loop with variable $\delta U^{k}$. This Newton loop contains a back-tracking line search to improve the convergence radius. Finally, the condition for the active set

$$
\left(B^{-1}\right)_{i i}\left(F-G \delta U_{h}^{k}\right)_{i}+c\left(\delta U_{h}^{k}\right)_{i}>0,
$$

reads using the notation from before:

$$
\left(B^{-1}\right)_{i i}\left(-\nabla E_{\varepsilon}\left(U_{h}^{k}\right)-\nabla^{2} E_{\varepsilon}\left(U_{h}^{k}\right) \delta U_{h}^{k}\right)_{i}+c\left(\delta U_{h}^{k}\right)_{i}>0 .
$$

We replace the linear residual $-\nabla E_{\varepsilon}\left(U_{h}^{k}\right)-\nabla^{2} E_{\varepsilon}\left(U_{h}^{k}\right) \delta U_{h}^{k}$ by the non-linear residual

$$
R\left(U_{h}^{k+1}\right)=-\nabla E_{\varepsilon}\left(U_{h}^{k+1}\right) .
$$

Remark 3.2. Because we merge two Newton iterations it is no longer correct to just require $\delta U_{h}^{k} \leq 0$ (point-wise) in each step since an intermediate active set allows a temporary violation of the crack growth condition during the Newton iteration. Therefore, we replace this condition with

$$
U_{h}^{k}+\delta U_{h}^{k} \leq U_{h}^{o l d}
$$

where $U_{h}^{\text {old }}$ is the solution of the last time step.

This gives us the algorithm:

Algorithm 3.2. Repeat for $k=0, \ldots$ until the active set $\mathcal{A}_{k}$ does not change and $\widetilde{R}\left(U_{h}^{k}\right)<$ TOL:

1. Assemble residual $R\left(U_{h}^{k}\right)$

2. Compute active set $\mathcal{A}_{k}=\left\{i \mid\left(B^{-1}\right)_{i i}\left(R_{k}\right)_{i}+c\left(\delta U_{h}^{k}\right)_{i}>0\right\}$

3. Assemble matrix $G=\nabla^{2} E_{\varepsilon}\left(U_{h}^{k}\right)$ and right-hand side $F=-\nabla E_{\varepsilon}\left(U_{h}^{k}\right)$

4. Eliminate rows and columns in $\mathcal{A}_{k}$ from $G$ and $F$ to obtain $\widetilde{G}$ and $\widetilde{F}$

5. Solve linear system $\widetilde{G} \delta U_{k}=\widetilde{F}$, i.e, find $\delta U_{h}^{k} \in V_{h} \times W_{h}$ with

$$
\nabla^{2} E_{\varepsilon}\left(U_{h}^{k}\right)\left(\delta U_{h}^{k}, \Psi\right)=-\nabla E_{\varepsilon}\left(U_{h}^{k}\right)(\Psi) \quad \forall \Psi \in V_{h} \times W_{h},
$$

where $\nabla^{2} E_{\varepsilon}$ and $\nabla E_{\varepsilon}$ are defined in Section 3.5 and 3.6.

6 . Find a step size $0<\omega \leq 1$ using line search to get

$$
U_{h}^{k+1}=U_{h}^{k}+\omega \delta U_{h}^{k},
$$

with $\widetilde{R}\left(U_{h}^{k+1}\right)<\widetilde{R}\left(U_{h}^{k}\right)$.

Remark 3.3. It is worth pointing out, that the residual $\widetilde{R}\left(U_{h}^{k}\right)$ might be far below the desired tolerance, however the active set can still change. Therefore, it is important to achieve both stopping criteria simultaneously:

$$
\mathcal{A}_{k+1}=\mathcal{A}_{k} \quad \text { and } \quad \widetilde{R}\left(U_{h}^{k}\right)<\mathrm{TOL} .
$$


Remark 3.4. It is important to distinguish between the full residual $R\left(U_{h}^{k}\right)$ and $\widetilde{R}\left(U_{h}^{k}\right)$. The latter is the residual on the inactive set, which can be computed by eliminating the active set constraints from the former.

Remark 3.5. To address directly the numerical solution of the non-convex energy functional by minimization, the alternate minimization algorithm with backtracking was suggested in $[3,27]$. Here, it is utilized by noting that the energy functional is convex in each single variable when the other is kept fixed. The full convergence proof can be found in [28].

\subsection{A fully-coupled formulation of the Euler-Lagrange equations}

For solving the forward PDE problem, we focus on a monolithic scheme in which all equations are solved simultaneously resulting in one semi-linear form. However, it is well known that the energy functional (3) is not convex simultaneously in both solution variables $\boldsymbol{u}$ and $\varphi$; but separately in each variable while keeping the other fixed. Consequently, solving the Euler-Lagrange equations in a straightforward way is not possible and influences the robustness of the solution scheme because of a (possibly) indefinite Hessian matrix $G$. The critical terms are the cross terms $\left(\left((1-\kappa) \varphi^{2}+\kappa\right) \sigma^{+}(\boldsymbol{u}), e(\boldsymbol{u})\right)$ and $\left(\varphi^{2} p\right.$, div $\left.\boldsymbol{u}\right)$. In this work, we linearize by linear extrapolation and time-lagging $\varphi \approx \tilde{\varphi}:=\tilde{\varphi}\left(\varphi^{n-1}, \varphi^{n-2}\right)$ in appropriate terms in order to obtain a convex energy functional. Here, $\varphi^{n-1}, \varphi^{n-2}$ denote the solutions to previous time steps.

Proposition 3.3. The energy functional $E_{\varepsilon}(\boldsymbol{u}, \tilde{\varphi})(3)$ with the fixed $\tilde{\varphi}$ is convex.

Proof. Applying a fixed, extrapolated $\varphi \approx \tilde{\varphi}\left(\varphi^{n-1}, \varphi^{n-2}\right)$ yields the energy functional $E_{\varepsilon}(\boldsymbol{u}, \tilde{\varphi})(3)$ instead of $E_{\varepsilon}(\boldsymbol{u}, \varphi)$. Without the term $\left(\varphi^{2} p, \operatorname{div} \boldsymbol{u}\right)$, the convexity of the energy functional is obvious and widely discussed in the literature. Adding $\left(\tilde{\varphi}^{2} p, \operatorname{div} \boldsymbol{u}\right)$ keeps the convexity because $\boldsymbol{u}$ is linear.

Remark 3.6. We note that in solving the variational Euler-Lagrange equations, a critical point (that is a global or local minimum or even a saddle point) is computed. However, the objective in minimizing a (non-convex) energy functional is to obtain a global minimum. We note that it might be physically questionable to achieve this. Additionally, the numerical techniques based on the alternate minimization algorithm with backtracking (fixing one variable while solving for the other in an iteration process) does not guarantee convergence to the global minimum $[27,3,8,28]$. In particular, the latter two papers provide discussions when relating energy minimization to the variational formulation in the presence of adaptive finite element discretizations. Considering the non-convex functional (3) without a priori linearization, we refer the reader to [29] for additional algorithms in the context of non-convex optimization.

Our strategy is as follows: In order to derive a monolithically-coupled variational formulation, we first assume $\varphi$ as given in (3) to obtain the Euler-Lagrange equations. In the first equation (i.e., the Euler-Lagrange equation with respect to $\boldsymbol{u}$ ), we replace $\varphi$ by an extrapolation $\tilde{\varphi}$. This results in a Jacobian (i.e., 
the Hessian of the energy functional (3)) that has a triangular block structure with elliptic terms on the diagonal, which can be solved using Galerkin finite elements. This treatment is shown in subsequent examples to be numerically robust. We are aware that the theoretical validity of the extrapolation remains an open question, since we have not established regularity in time.

The formulation of the semi-linear problem then reads: Find $U:=\{\boldsymbol{u}, \varphi\} \in$ $V \times W$ such that

$$
\begin{aligned}
\nabla E_{\varepsilon}(U)(\Psi)= & \left(\left((1-\kappa) \tilde{\varphi}^{2}+\kappa\right) \sigma^{+}(\boldsymbol{u}), e(\boldsymbol{w})\right)+\left(\sigma^{-}(\boldsymbol{u}), e(\boldsymbol{w})\right)-(\alpha-1)\left(\tilde{\varphi}^{2} p, \operatorname{div} \boldsymbol{w}\right) \\
& +(1-\kappa)\left(\varphi \sigma^{+}(\boldsymbol{u}): e(\boldsymbol{u}), \psi\right)-2(\alpha-1)(\varphi p \operatorname{div} \boldsymbol{u}, \psi) \\
& +G_{c}\left(-\frac{1}{\varepsilon}(1-\varphi, \psi)+\varepsilon(\nabla \varphi, \nabla \psi)\right)=0 \quad \forall \Psi:=\{\boldsymbol{w}, \psi\} \in V \times W .
\end{aligned}
$$

The corresponding Jacobian is built by computing the directional derivative $A^{\prime}(U)(\delta U, \Psi)$. Then, $\delta U:=\{\delta \mathbf{u}, \delta \varphi\} \in V \times W$ such that

$$
\begin{aligned}
\nabla^{2} E_{\varepsilon}(U)(\delta U, \Psi)= & \left(\left((1-\kappa) \tilde{\varphi}^{2}+\kappa\right) \sigma^{+}(\delta \mathbf{u}), e(\boldsymbol{w})\right)+\left(\sigma^{-}(\delta \mathbf{u}), e(\boldsymbol{w})\right) \\
& +(1-\kappa)\left(\delta \varphi \sigma^{+}(\boldsymbol{u}): e(\boldsymbol{u})+2 \varphi \sigma^{+}(\delta \mathbf{u}): e(\boldsymbol{u}), \psi\right) \\
& -2(\alpha-1) p(\delta \varphi \operatorname{div} \boldsymbol{u}+\varphi \operatorname{div} \delta \mathbf{u}, \psi) \\
& +G_{c}\left(\frac{1}{\varepsilon}(\delta \varphi, \psi)+\varepsilon(\nabla \delta \varphi, \nabla \psi)\right)=0 \quad \forall \Psi:=\{\boldsymbol{w}, \psi\} \in V \times W .
\end{aligned}
$$

In $\sigma^{+}(\delta \mathbf{u})$ and $\sigma^{-}(\delta \mathbf{u})$ we employ the derivative of $e^{+}$, which is given by

$$
e^{+}(\delta \mathbf{u})=P(\delta \mathbf{u}) \Lambda^{+} P^{T}+P \Lambda^{+}(\delta \mathbf{u}) P^{T}+P \Lambda^{+} P^{T}(\delta \mathbf{u}) .
$$

3.6. Spatial discretization and block structure of the Euler-Lagrange linear equation system

In this section, we consider the structure and solution of the linear discrete system (15) arising in each Newton step. For spatial discretization, we use the previously introduced spaces $V_{h} \times W_{h}$ with vector valued basis

$$
\left\{\psi_{i} \mid i=1, \ldots, N\right\},
$$

where the basis functions are primitive (they are only non-zero in one component), so we can separate them into displacement and phase-field basis functions and sort them accordingly:

$$
\begin{aligned}
\psi_{i} & =\left(\begin{array}{c}
\chi_{i}^{u} \\
0
\end{array}\right), \text { for } i=1, \ldots, N_{u}, \\
\psi_{\left(N_{u}+i\right)} & =\left(\begin{array}{c}
0 \\
\chi_{i}^{\varphi}
\end{array}\right), \text { for } i=1, \ldots, N_{\varphi},
\end{aligned}
$$

where $N_{u}+N_{\varphi}=N$. This is now used to transform (15) into a system of the form

$$
M x=F,
$$


where $M$ is a block matrix (the Jacobian) and $F$ the right hand side consisting of the residuals. The block structure is

$$
M=\left(\begin{array}{ll}
M^{u u} & M^{u \varphi} \\
M^{\varphi u} & M^{\varphi \varphi}
\end{array}\right), \quad F=\left(\begin{array}{c}
F^{u} \\
F^{\varphi}
\end{array}\right),
$$

with entries coming from (17):

$$
\begin{aligned}
M_{i, j}^{u u}= & \left(\left((1-\kappa) \tilde{\varphi}^{2}+\kappa\right) \sigma^{+}\left(\chi_{j}^{u}\right), e\left(\chi_{i}^{u}\right)\right)+\left(\sigma^{-}\left(\chi_{j}^{u}\right), e\left(\chi_{i}^{u}\right)\right), \\
M_{i, j}^{\varphi u}= & 2(1-\kappa)\left(\varphi \sigma^{+}\left(\chi_{j}^{u}\right): e(\boldsymbol{u}), \chi_{i}^{\varphi}\right)-2(\alpha-1) p\left(\varphi \operatorname{div}\left(\chi_{j}^{u}\right), \chi_{i}^{\varphi}\right), \\
M_{i, j}^{u \varphi}= & 0, \\
M_{i, j}^{\varphi \varphi}= & (1-\kappa)\left(\sigma^{+}(\boldsymbol{u}): e(\boldsymbol{u}) \chi_{j}^{\varphi}, \chi_{i}^{\varphi}\right)-2(\alpha-1) p\left(\operatorname{div}(\boldsymbol{u}) \chi_{j}^{\varphi}, \chi_{i}^{\varphi}\right) \\
& +G_{c}\left(\frac{1}{\varepsilon}\left(\chi_{j}^{\varphi}, \chi_{i}^{\varphi}\right)+\varepsilon\left(\nabla \chi_{j}^{\varphi}, \nabla \chi_{i}^{\varphi}\right)\right) .
\end{aligned}
$$

The right hand side consists of the corresponding residuals (see semi-linear form (16)). In particular, we have

$$
\begin{aligned}
F_{i}^{u}= & -\widetilde{A}\left(U_{k}\right)\left(\chi_{i}^{u}\right) \\
= & \left(\left((1-\kappa) \tilde{\varphi}_{k}^{2}+\kappa\right) \sigma^{+}\left(\boldsymbol{u}_{k}\right), e\left(\chi_{i}^{u}\right)\right)+\left(\sigma^{-}\left(\boldsymbol{u}_{k}\right), e\left(\chi_{i}^{u}\right)\right)-(\alpha-1)\left(\tilde{\varphi}_{k}^{2} p, \operatorname{div} \chi_{i}^{u}\right), \\
F_{i}^{\varphi}= & -\widetilde{A}\left(U_{k}\right)\left(\chi_{i}^{\varphi}\right)=(1-\kappa)\left(\varphi_{k} \sigma^{+}\left(\boldsymbol{u}_{k}\right): e\left(\boldsymbol{u}_{k}\right), \chi_{i}^{\varphi}\right)-2(\alpha-1)\left(\varphi_{k} p \operatorname{div} \boldsymbol{u}_{k}, \chi_{i}^{\varphi}\right) \\
& +G_{c}\left(-\frac{1}{\varepsilon}\left(1-\varphi_{k}, \chi_{i}^{\varphi}\right)+\varepsilon\left(\nabla \varphi_{k}, \nabla \chi_{i}^{\varphi}\right)\right) .
\end{aligned}
$$

In the matrix, the degrees of freedom that belong to Dirichlet conditions (here only displacements since we assume Neumann conditions for the phase-field) are strongly enforced by replacing the corresponding rows and columns as usual in a finite element code. In a similar fashion, the rows and columns that belong to nodes of the active set are removed from the matrix. Corresponding right hand side values in the vector $F$ are set to zero. This is exactly Step 4 in Algorithm 3.2. The linear system (18) is solved with appropriate solvers in Trilinos [30].

Remark 3.7. Since we replaced $\varphi^{2}$ by $\tilde{\varphi}^{2}$ the block $M_{i, j}^{u \varphi}$ is zero and the Jacobian $G$ has triangular structure. In the other case, all blocks would be nonzero.

\section{A predictor-corrector scheme for mesh adaptivity}

This second key section is concerned with local mesh adaptivity. Phasefield approaches require fine meshes around the interface (here, the fracture) in order to provide solutions of sufficient accuracy. There are several studies that have been investigated anisotropies introduced by the mesh [31], anisotropic adaptive mesh refinement [7], and prerefined meshes when the crack path is known a priori [6]. Here, we are interested in a general treatment in which no a priori information of the crack path is required. 


\subsection{Properties of the algorithm}

The algorithm asks for the following properties:

- Keep a single fixed, small $\varepsilon$ during the entire computation. Decreasing $\varepsilon$ (locally) during the computation will not allow for an increase in accuracy of the solution. While reducing $\varepsilon$ would result in a thinner crack mushy zone, the irreversibility constraint would not allow for this to happen. As a consequence, $\varepsilon$ should not be changed during the computation.

Note that it would be possible to increase $\varepsilon$ locally in order to allow the mesh to be locally coarsened in order to reduce computational effort; this study was not investigated in this paper. However, locally varying $\varepsilon$ would result in a changing crack mushy zone, which is undesirable.

- Ensure $h<\varepsilon$ inside crack region. It is required to have a sufficiently small mesh size $h$ to resolve the transition of the phase field variable. The width of this zone is controlled by the choice of $\varepsilon$. Importantly, the $\varepsilon$ - $h$ relationship is only required to be satisfied inside or directly around the current crack region and not in the whole computational domain.

\section{- Error is controlled by $\varepsilon$, not $h$.}

In contrast to standard a-posteriori or goal oriented adaptive mesh refinement, just refining the mesh does not reduce the discretization error significantly. This is because the choice of $\varepsilon$ determines the width of the mushy zone around the crack path. Ideally, an adaptive method would try to minimize $\varepsilon$ and pick an appropriate $h$ to minimize the discretization error introduced by the mesh size.

- No requirement of prior knowledge about crack location(s). Typically, especially for more realistic problems, the final location of the cracks is unknown. While it is an option to repeat the whole computation on a finer mesh that is determined using the first computation, this is too expensive to be practical. Therefore, the algorithm should detect during the computation in which direction the cracks are growing.

- Handling fast growing cracks. Only adapting the mesh based on the current crack location before moving on to the next time step may result that the adaptive mesh lags behind in time and does not resolve the crack region adequately, especially if the crack is growing rapidly.

Our proposed algorithm works as follows: We first pick a single, small $\varepsilon$, and then decide on an adaptive refinement level $r$ for the crack region that ensures $h<\varepsilon$. We then refine the mesh adaptively during the computation so that it is on level $r$ in the crack region. To handle fast growing cracks with a priori unknown paths, we employ a predictor-corrector scheme that keeps repeating the current time step to guarantee the finest mesh level $r$ in the crack region. 
Figure 1: Predictor-corrector scheme: 1. advance in time, crack leaves fine mesh. 2. refine and go back in time (interpolate old solution). 3. advance in time on new mesh. Repeat until mesh doesn't change anymore. Refinement is triggered for $\varphi<C=0.2$ (green contour line) here.

\subsection{The algorithm in a single time step}

Let the solution to time step $t^{n}$ be given; Figure 1 top left. The full problem is solved with a prediction of the new crack path at time step $t^{n+1}$; Figure 1 top right. If the crack is not resolved adequately, we employ a predictor-corrector cycle: First, we refine the mesh based on the new solution and interpolate the old solution (at $t^{n}$ ) onto the new mesh (Figure 1, bottom left). The refinement is done using a chosen threshold $0<C<1(C=1$ corresponds to global mesh refinement) for the phase-field $\varphi$. Each cell that has at least one support point with value $\varphi\left(x_{i}\right)<C$ will be refined unless we are already at the maximum desired refinement level $r$. Then we solve for the solution at $t^{n+1}$ again, but on the refined mesh (Figure 1, bottom right). This process is repeated until the mesh does not change anymore.

The cycle always terminates in a finite number of steps because a fixed maximum refinement level is chosen. In practice, predictor-corrector cycles only happen when the crack is rapidly growing and, at least in our experiments, terminate after 1 to 3 cycles. As a result this guarantees $\{x: \varphi(x)<C\} \subset \Omega$ is resolved with the maximum desired refinement level.

Of course, the price to pay is are additional systems to solve to predict the crack path but we nevertheless obtain a highly efficient method since it allows us to grow the mesh (i.e., the degrees of freedom) together with the crack. 


\subsection{The final predictor-corrector algorithm for local mesh adaptivity}

In summary, our proposed predictor-corrector scheme - forcing the growing crack region to always be resolved with a fine mesh - reads:

Algorithm 4.1. Choose a fixed refinement level $r$ for the crack region. On level $r$, determine $h_{\max }^{(r)}$ and pick an appropriate $\varepsilon:=\varepsilon^{(r)}>h_{\max }^{(r)}$. Select a bound $0<C<1$ for $\varphi$ to be considered inside the crack. For each time step do:

1. Solve for solution $\left(u^{n+1}, \varphi^{n+1}\right)$ at $t_{n+1}$.

2. If cells need to be refined (cell with level $k<r$ has $\varphi^{n+1}(x)<C$ ): refine and transfer solution from $t_{n}$, goto 1 .

Remark 4.1. The parameter $\varepsilon$ needs to chosen relative to the largest cell size $h$ that can appear on level $r$ during the computation. For refinement of a quadrilateral mesh this quantity can be computed from the set of coarse cells $\mathcal{T}$ using

$$
h_{\max }^{(r)}=\max _{T \in \mathcal{T}} 2^{-r} h_{T}
$$

where $h_{T}$ is the size of cell $T$.

Remark 4.2. Another interpretation of this scheme is that we pick a-priori constant values $\varepsilon$ and $h$ for the computation and then coarsen cells away from the crack region where the solution is smooth.

\section{Numerical Tests}

In this final section, we present several crack propagation scenarios in pure elasticity and pressurized regimes. In all tests, the focus is on a robust and cost efficient solution to study convergence of certain quantities of interest and features such as joining and branching of cracks. Specifically in Example 1, Sneddon's [32] pressurized crack benchmark is considered. In the second subsection (Examples 2 and 3), we focus on well-known tests in elasticity in mechanical engineering; namely, the single edge notched tension and shear tests; and then furthermore in Example 4 on a very classical benchmark, the so-called symmetric three point bending test. In the final tests (Examples 5 and 6 ), we treat two growing pressurized fractures in homogeneous and heterogeneous elastic media.

\subsection{Quantities of interest}

In the first test, we consider the evaluation of the crack opening displacement (COD; also known as aperture):

$$
C O D=\int_{0}^{4} \boldsymbol{u}\left(x_{0}, y\right) \cdot \nabla \varphi\left(x_{0}, y\right) d y
$$


where $\varphi$ is as before our phase-field function and $x_{0}$ the $x$-coordinate of the integration line. The analytical solution for the crack opening displacement derived by Sneddon and Lowengrub [32] is given by:

$$
C O D=2 \frac{p l_{0}}{E^{\prime}}\left(1-\frac{x^{2}}{l_{0}^{2}}\right)^{1 / 2}=3.84 \times 10^{-4}\left(1-\frac{x^{2}}{l_{0}^{2}}\right)^{1 / 2},
$$

where $E^{\prime}=\frac{E}{1-\nu^{2}}$ with $p$ (applied pressure), and the half crack length $l_{0}$. All concrete values are specified below.

In the other tests, we compute load functionals over parts $\Gamma$ of the boundary, i.e,

$$
\tau=\left(F_{x}, F_{y}\right):=\int_{\Gamma} \sigma(\boldsymbol{u}) \mathbf{n} d s .
$$

In addition, we compute the bulk energy $E_{B}$ given by

$$
E_{B}=\int_{\Omega}\left([1-\kappa] \varphi^{2}+\kappa\right) \psi(e) d x
$$

and crack energy

$$
E_{C}=\frac{G_{c}}{2} \int_{\Omega}\left(\frac{(\varphi-1)^{2}}{\varepsilon}+\varepsilon|\nabla \varphi|^{2}\right) d x
$$

with the strain energy functional

$$
\psi(e):=\mu \operatorname{tr}\left(e(\boldsymbol{u})^{2}\right)+\frac{1}{2} \lambda \operatorname{tr}(e(\boldsymbol{u}))^{2},
$$

the previously introduced symmetric strain tensor

$$
e:=e(\boldsymbol{u}):=\frac{1}{2}\left(\nabla \boldsymbol{u}+\nabla \boldsymbol{u}^{T}\right)
$$

and $|\nabla \varphi|^{2}:=\nabla \varphi: \nabla \varphi$

\subsection{Programming code}

The programming software is based on deal.II $[33,34]$ and specifically on the adaptation and extension of the template for solving fully-coupled multiphysics problems [35] combined with the implementation of the primal-dual active set strategy from deal.II's step-41 [36].

\subsection{D Sneddon test with pressurized fracture (Example 1)}

The first example is based on the theoretical calculations of [32, 37]. Specifically, we consider a 2D problem in which all data are given in dimensionless form; where a (constant) pressure $p=10^{-3}$ is used to drive the deformation and crack width evolution. The configuration is the same as explained in [38] and illustrated in Figure 2. Therefore, we deal with the following geometric data: $\Omega=(0,4)^{2}$ and a (prescribed) initial crack with length $l_{0}=0.4$ on 
$\Omega_{C}=(1.8-h, 2.2+h) \times(2-h, 2-h) \subset \Omega$. As boundary conditions, we set the displacements to zero on $\partial \Omega$.

The fracture toughness is chosen as $G_{c}=1.0$. The mechanical parameters are Young's modulus and Poisson's ration $E=1.0$ and $\nu_{s}=0.2$. The relationship to the Lamé coefficients $\mu_{s}$ and $\lambda_{s}$ is given by:

$$
\mu_{s}=\frac{E}{2\left(1+\nu_{s}\right)}, \quad \lambda_{s}=\frac{\nu_{s} E_{s}}{\left(1+\nu_{s}\right)\left(1-2 \nu_{s}\right)} .
$$

The regularization parameters are chosen as $\varepsilon=2 h$ and $\varepsilon=0.5 h^{0.5}$; and $\kappa=$ $10^{-10}$. This test case is computed in a quasi-stationary manner: that is, we solve several pseudo-time steps until the residual $<\mathrm{TOL}=10^{-5}$ is reached. The goals of this test are to observe the crack opening displacement, and in particular to show the crack tip approximation.

Our findings are summarized in Figure 3 where we show the crack opening displacement for a sequence of locally refined meshes. In addition, observing the crack tips at 1.8 and 2.2 demonstrates convergence towards Sneddon's manufactured solution. We remark that the $\varepsilon$ - $h$ choice is of minor importance in this example since in both computations similar crack opening values are obtained.
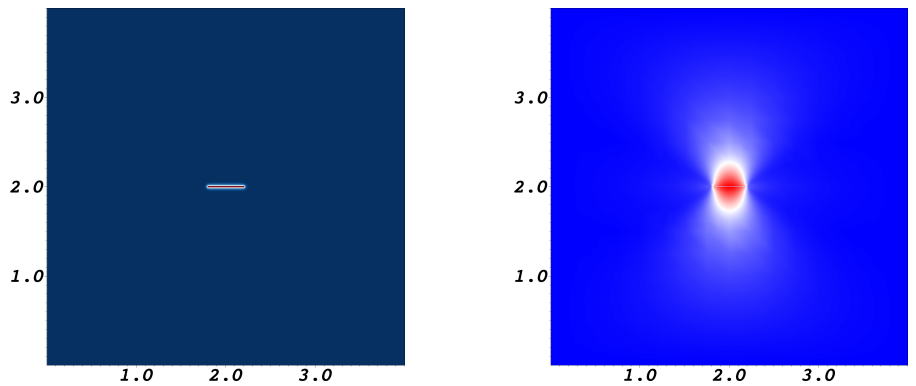

Figure 2: Example 1: crack location (in red) and magnitude (highest in red) of the displacements.
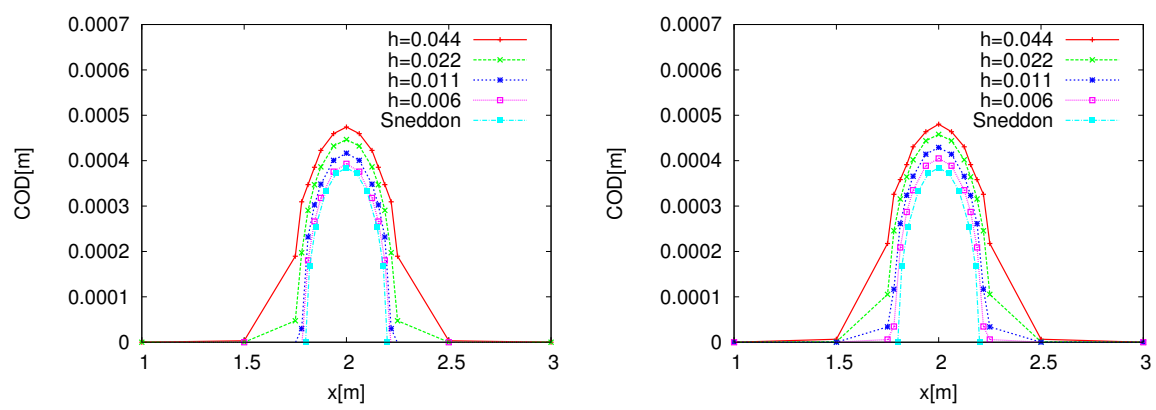

Figure 3: Example 1: crack opening displacement for $\varepsilon=2 h$ (left) and $\varepsilon=0.5 h^{0.5}$ (right). In particular we point the reader to the excellent approximation of the crack tips under spatial mesh refinement. 


\subsection{Single edge notched tension and shear tests (Examples 2 and 3)}

In this example, we restrict our attention to pure elastic crack-propagation examples in order to test our solver for cases with unstable and brutal crack growth. Specifically, we show that dynamic crack-oriented mesh adaptivity drastically reduces the computational cost.

Similar settings have been experimentally studied and it is well known that under constant tension the crack grows straight, while under constant shear forces the crack grows in a curve towards a corner. Moreover, we point out that the crack irreversibility condition is redundant for Example 2 and we obtain the same results as Miehe et al. [5] and Borden et al. [6].

The geometric and material properties are the same as used in [5]. The configuration is displayed in Figure 4.
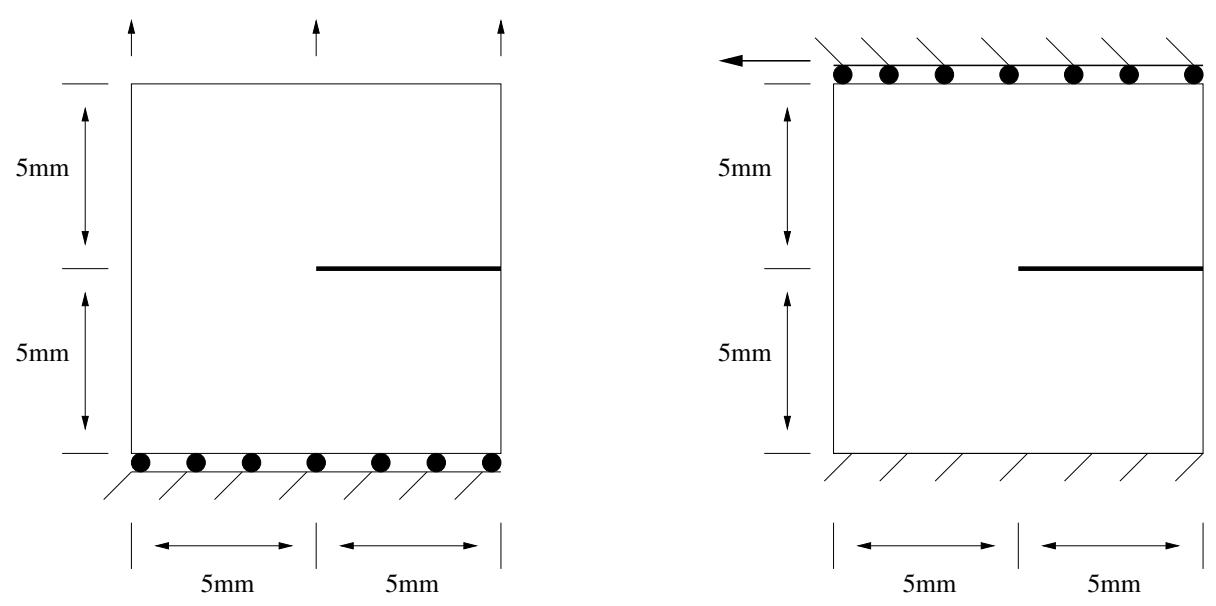

Figure 4: Example 2: Single edge notched tension test (Example 2; left) and shear test (Example 3; right). In detail, the boundary conditions are for Example 2: $\boldsymbol{u}_{y}=0 \mathrm{~mm}$ (homogeneous Dirichlet) and traction free (homogeneous Neumann conditions) in $x$-direction on the bottom. On the top boundary $\Gamma_{t o p}$, we prescribe $\boldsymbol{u}_{x}=0 m m$ and $\boldsymbol{u}_{y}$ as provided in (22). All other boundaries including the slit are traction free (homogeneous Neumann conditions). For Example 3, we prescribe the following conditions: On the left and right boundaries, $\boldsymbol{u}_{y}=0 \mathrm{~mm}$ and traction-free in $x$-direction. On the bottom part, we use $\boldsymbol{u}_{x}=\boldsymbol{u}_{y}=0 \mathrm{~mm}$ and on $\Gamma_{t o p}$, we prescribe $\boldsymbol{u}_{y}=0 \mathrm{~mm}$ and $\boldsymbol{u}_{x}$ as stated in (23). Finally, the lower part of the slit is fixed in $y$-direction, i.e., $\boldsymbol{u}_{y}=0 \mathrm{~mm}$.

Specifically, we use $\mu=80.77 \mathrm{kN} / \mathrm{mm}^{2}, \lambda=121.15 \mathrm{kN} / \mathrm{mm}^{2}$, and $G_{c}=$ $2.7 \mathrm{~N} / \mathrm{mm}$. The crack growth is driven by a non-homogeneous Dirichlet condition for the displacement field on $\Gamma_{t o p}$, the top boundary of $\Omega$. We increase the displacement on $\Gamma_{\text {top }}$ over time, namely we apply non-homogeneous Dirichlet conditions:

$$
\begin{array}{ll}
\boldsymbol{u}_{y}=t \overline{\boldsymbol{u}}, & \overline{\boldsymbol{u}}=1 \mathrm{~mm} / \mathrm{s} \text { (Example 2) } \\
\boldsymbol{u}_{x}=t \overline{\boldsymbol{u}}, & \overline{\boldsymbol{u}}=1 \mathrm{~mm} / \mathrm{s} \text { (Example } 3)
\end{array}
$$


where $t$ denotes the total time. Furthermore, we set $k=10^{-10}[\mathrm{~mm}]$ and $\varepsilon=$ $2 h[\mathrm{~mm}]$ for the first test. For the second test the $h-\varepsilon$ relation is provided below. We evaluate the surface load vector on the $\Gamma_{\text {top }}$ as

$$
\tau=\left(F_{x}, F_{y}\right):=\int_{\Gamma_{t o p}} \sigma(\boldsymbol{u}) \mathbf{n} d s,
$$

with normal vector $\mathbf{n}$, and we are particularly interested in $F_{y}$ for Example 2 and $F_{x}$ for Example 3. Our findings for the surface load evolution are provided in Figure 5 and the corresponding crack patterns are displayed in Figure 6. Specifically, the approach is stable with respect to spatial mesh refinement.
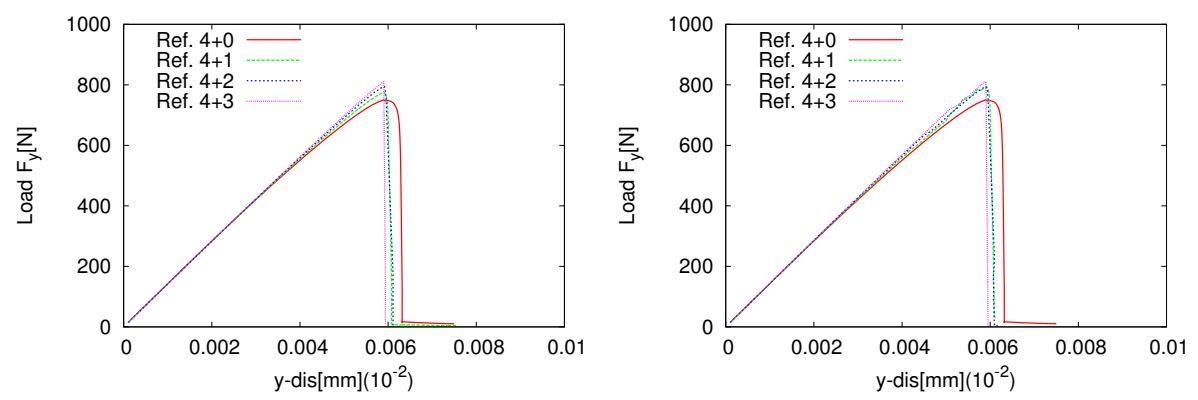

Figure 5: Example 2: Single edge notched tension test. Left: we locally pre-refine the region where we expect the crack to grow. On the right the mesh is dynamically refined using the phase-field variable. Both approaches lead to identical results. 


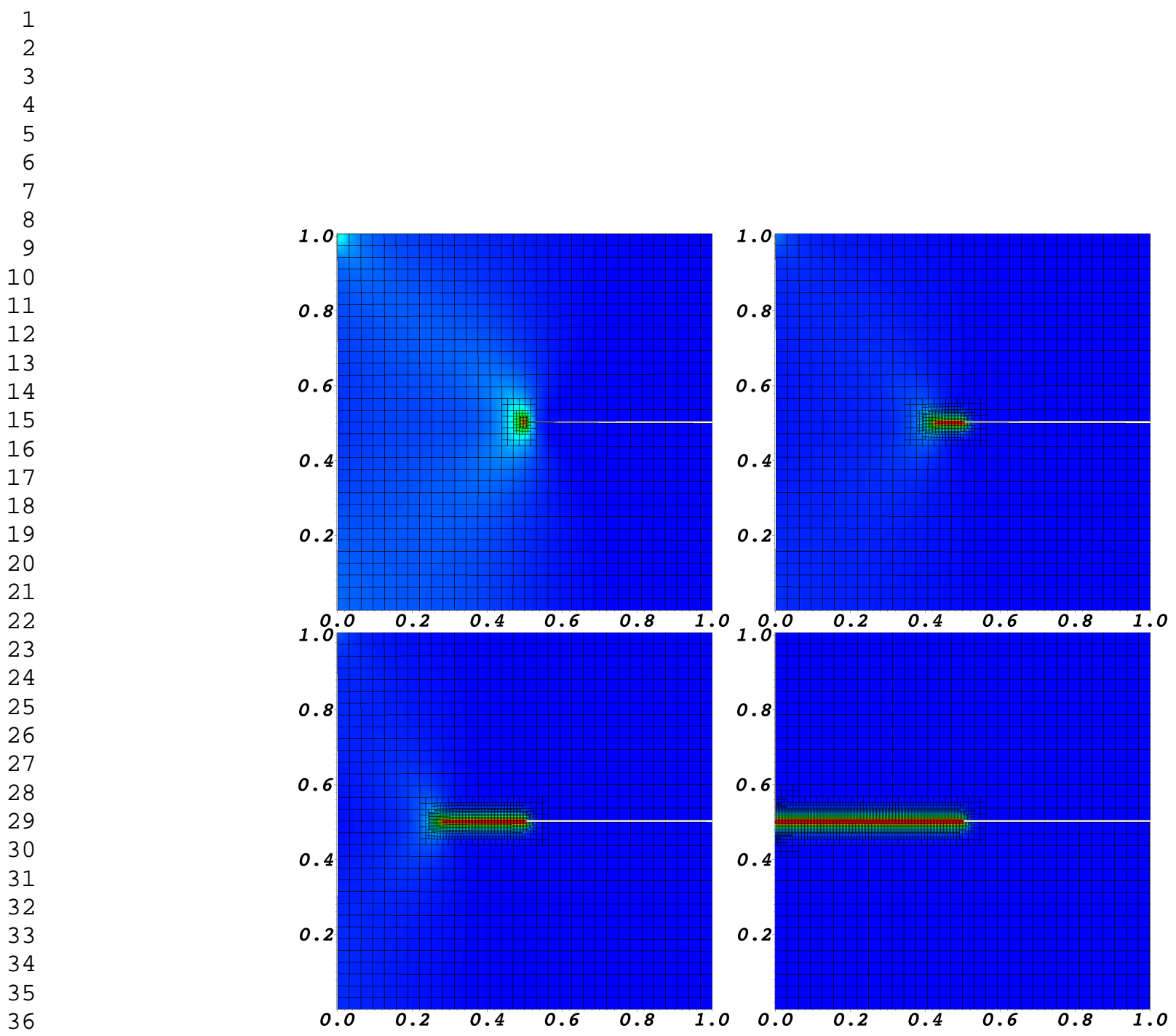

Figure 6: Example 2: Single edge notched tension test. Crack propagation in red and dynamic mesh refinement at different times (going from top left to bottom right). 
Single edge notched shear test

In the single edge notched shear test, it is important to consider the correct boundary conditions and the spectral decomposition of the strain energy $e(\boldsymbol{u})$ into tensile $e^{+}(\boldsymbol{u})$ and compressive parts $e^{-}(\boldsymbol{u})$. We refer to [4] for a detailed physical motivation. Moreover, this test is useful in addressing whether the crack follows the mesh or vice versa. A motivation in studying this question is posed in [7]; in this regard, we also refer the reader to Section 5.3 in [38] and to Section 5.5 below.

Our first aim, and most importantly, is to study $h$-refinement for fixed $\varepsilon$. These studies are performed on different mesh levels $4+0$ (with $h=0.044 \mathrm{~mm}$ ), $4+1($ with $h=0.022 \mathrm{~mm}), 4+2($ with $h=0.011 \mathrm{~mm}), 4+3($ with $h=0.0056 \mathrm{~mm})$, and $4+4$ (with $h=0.0027 \mathrm{~mm}$ ). In a second set of studies, we vary the loading step size $\delta t$. Thirdly, we consider model parameter refinements in $\varepsilon$ linked to spatial $h$ refinement; namely $h=o(\varepsilon)$. Here, we propose three scenarios Case 1: $\varepsilon=2 h[\mathrm{~mm}]$, Case 2: $\varepsilon=c h^{0.5}[\mathrm{~mm}], c=0.25$, and Case 3: $\varepsilon=c h^{0.25}[\mathrm{~mm}], c=$ 0.125 . In comparison, the reference values in [5] are obtained on an effective mesh size $h=0.002 \mathrm{~mm}$. Our reference load-displacements curves were obtained by interpolation from computational results provided in [5] and [6].

We draw the following conclusions from our findings. In Figure 7, we fix $h$ and study the convergence for different $\varepsilon$. The influence of its choice is significantly larger on fine meshes. Consequently, this test demonstrates that drawing any conclusions on coarse meshes might be redundant and fine meshes must be studied.
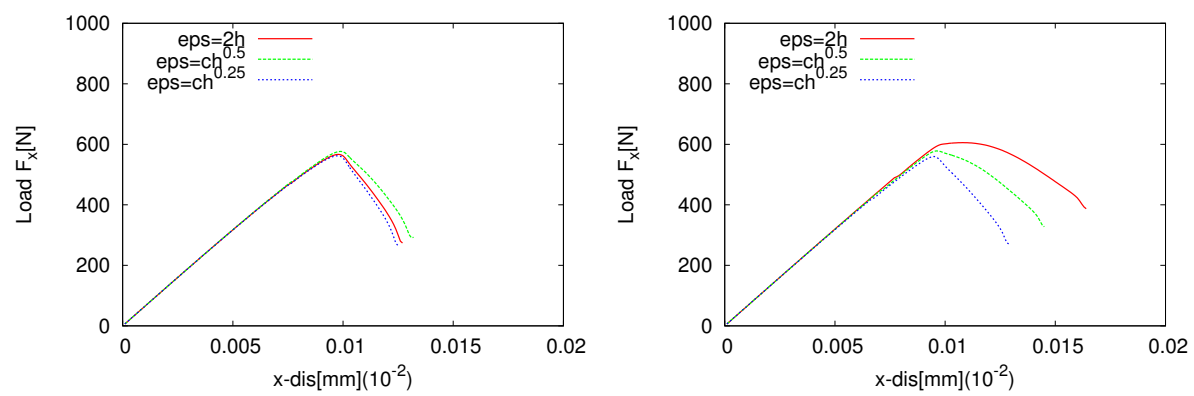

Figure 7: Example 3: Single edge notched shear test. Load-displacement curve on the coarsest $4+1$ (left) and finest meshes $4+3$ (right). Here, we compare convergence with respect to $\varepsilon$. The more the $\Gamma$ convergence requirement is fulfilled the better the convergence can be identified. This becomes more visible on the finer meshes.

Next, we fix $\varepsilon=0.088 \mathrm{~mm}\left(=2 h^{(4+0)}\right)$ in Case 1 and refine $h$ in order to study $h$ convergence. Our results are displayed in Figure 8 in which we clearly identify spatial convergence with respect to $h$. 


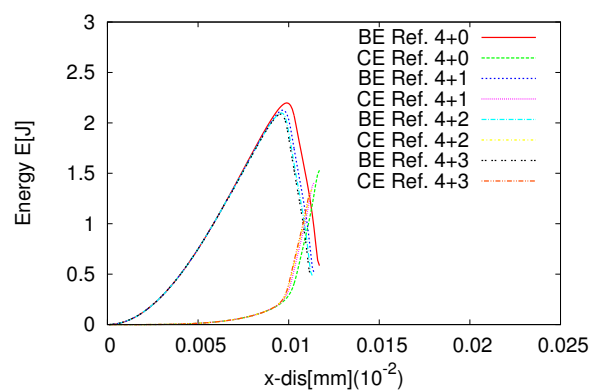

Figure 8: Example 3: Single edge notched shear test. Load-displacement curve (left) and bulk (BE) and crack (CE) energies (right) for fixed $\varepsilon=0.088 \mathrm{~mm}\left(=2 h^{(4+0)}\right)$ and refinement in $h$. Spatial convergence can be clearly observed.

In the next tests, $h$ and $\varepsilon$ are refined simultaneously. Case 1 shows poor spatial behavior for a fixed time step size $\delta t=10^{-4} s$ and is not reported here. Secondly, we compare a priori local refinement and predictor-corrector refinement, which yield similar findings (see Figure 12). From these results, we infer our proposed predictor-corrector refinement is correct. Furthermore, applying the theory of $\Gamma$ convergence; namely, cases 2 and 3, the loading curves are clustered together as shown in Figure 9. In fact, the better clustering is obtained for $\varepsilon=c h^{m}$ with small $m>0$ and $m \ll 1$. This is in agreement with the theoretical assertions of $\Gamma$-convergence telling us that $h=o(\varepsilon)$.
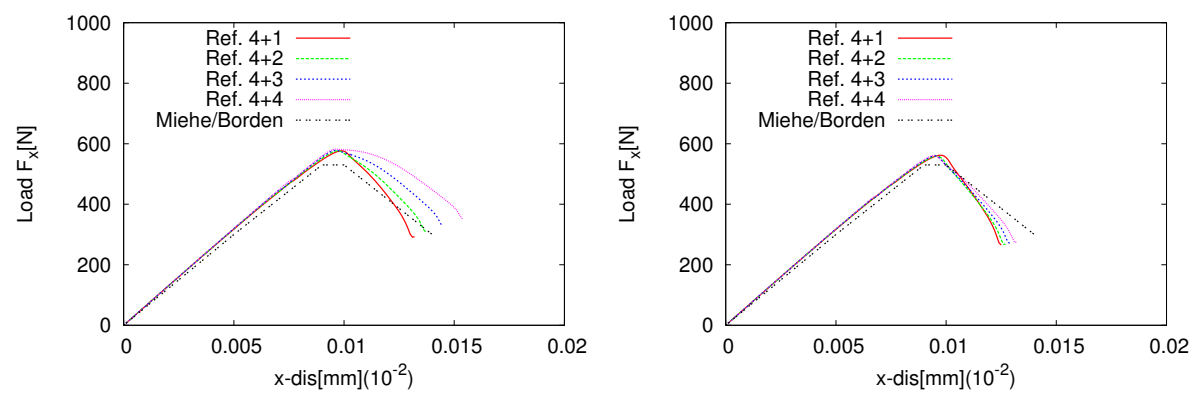

Figure 9: Example 3: Single edge notched shear test. Load-displacement curve for the Case 2 (left) and Case 3 (right). We observe that if we choose $h$ and $\varepsilon$ according to the theoretical requirement of $\Gamma$ convergence with $h=o(\varepsilon)$, then clustering of the load-displacements curves can be seen. We notice that this test using Case 1 leads to poor convergence.

Our findings in Figure 10 show the evolution of bulk (20) and crack energies (21) for Case 1 and Case 3. The results illustrate the expected behavior: As long as the crack does not grow (up to $t=0.0095 \mathrm{~s}$ ), there is only an increase in bulk energy. Once the crack starts growing, bulk energy is dissipated into crack energy. 

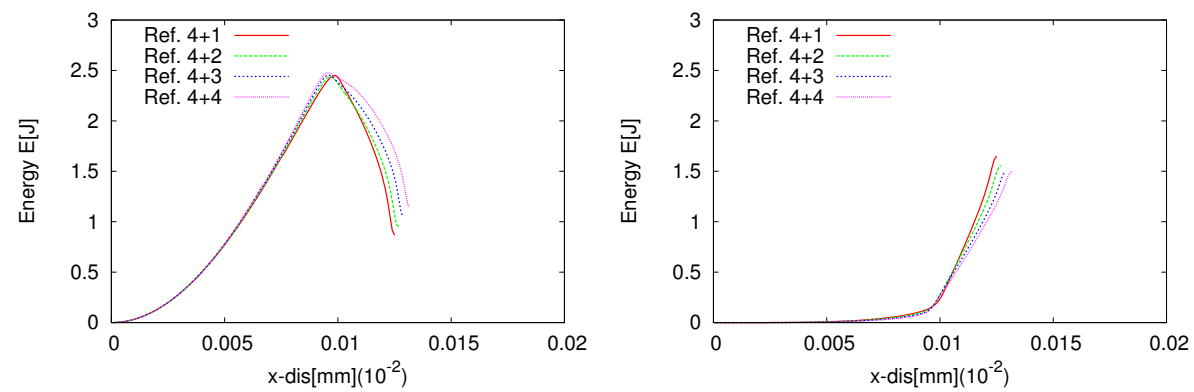

Figure 10: Example 2: Single edge notched shear test. Comparison of bulk (left) and crack (right) energies for Case 3. Once crack propagation starts, the bulk energy decreases and crack energy increases. In summary (like for the previously discussed load-displacement curves), we observe that the energy approximation clearly depends on the choice of the $h$ - $\varepsilon$-relation.

In the Figure 11, several tests studying the influence of the time step size choice are performed. Here, we confirm our previous observations (and also the theory) that irrespectively of the time step size, Case 1 shows poor spatial convergence; whereas in Case 3 spatial convergence is obtained for both choices $\delta t=10^{-5} s$ and $\delta t=10^{-6} s$.
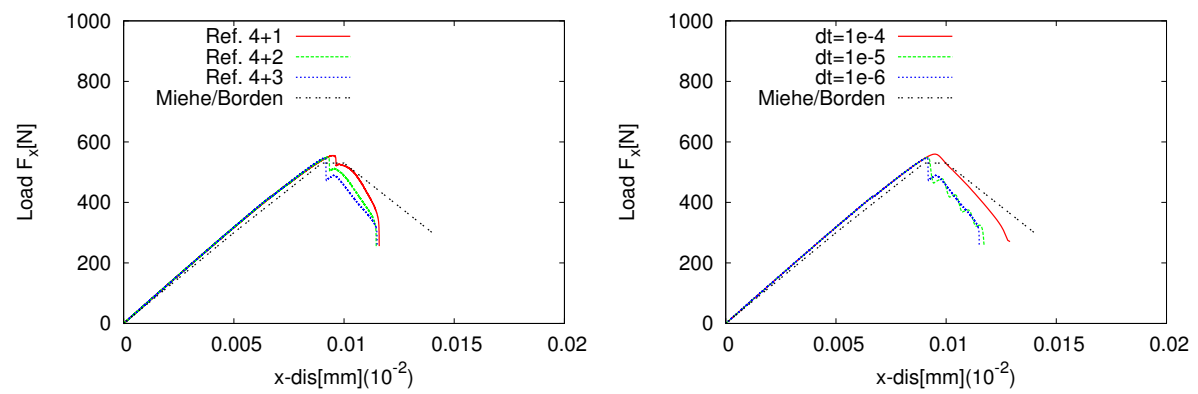

Figure 11: Example 2: Tests for Case 3 and smaller time steps $\delta t=10^{-6} s$ (left). In contrast to Case 1, we clearly identify spatial convergence for both times step choices. At right, Case 3 on $4+3$-refined meshes and convergence studies with respect to time. Fixing the spatial mesh level, we observe time convergence for both choices $\varepsilon=2 h$ as well as $\varepsilon=c h^{0.25}$ with $c=0.125$.

Despite the fact that we have not established theoretically the time convergence, our computational results demonstrate temporal convergence as observed in Figure 11. Here, three different time step sizes on the finest spatial mesh level clearly demonstrate convergence in time. 


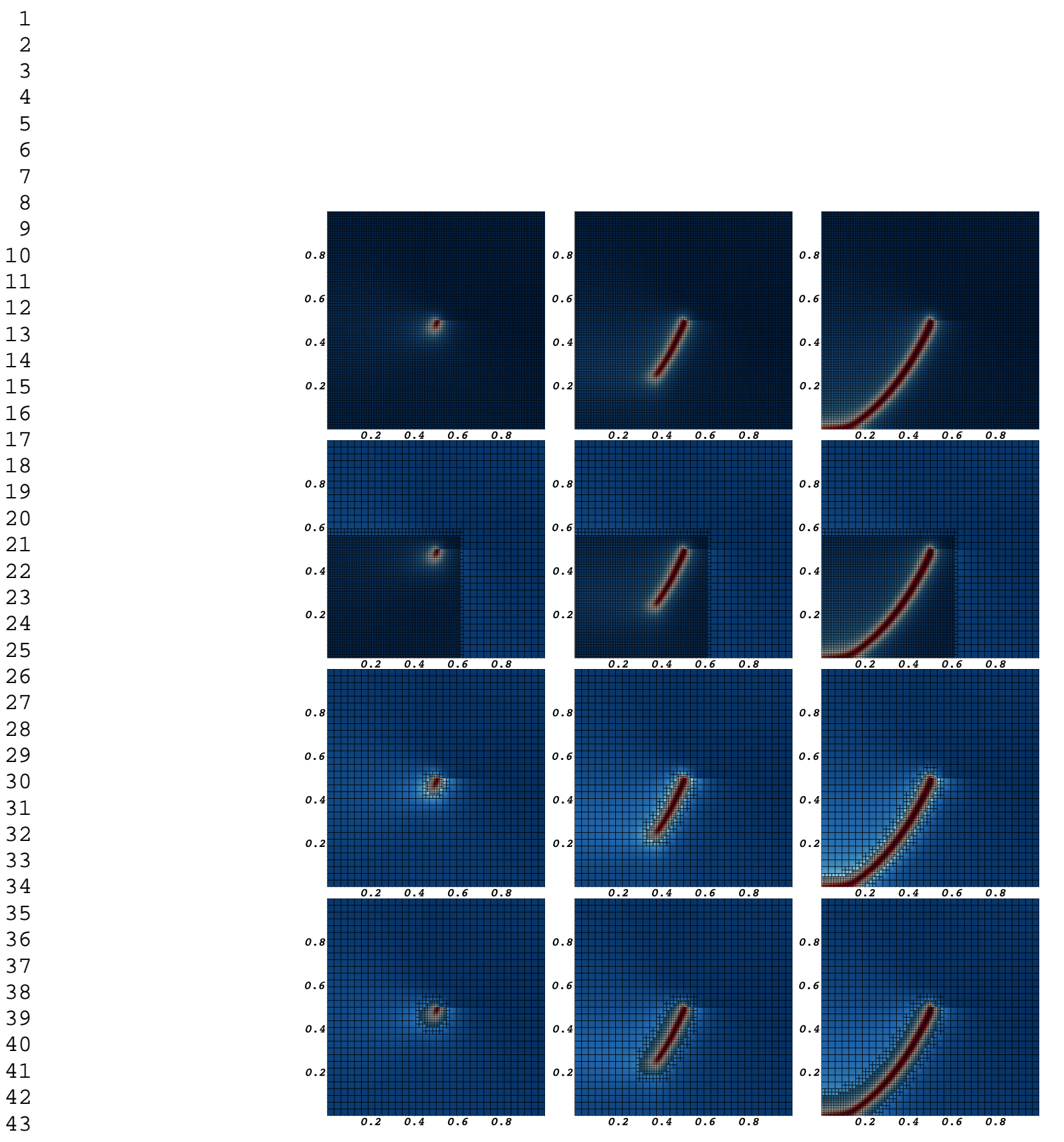

Figure 12: Example 3: Single edge notched shear test for $4+2$-refined meshes. Crack propagation in red and dynamic mesh refinement at different times $T=100 \mathrm{~s}, 120 \mathrm{~s}, 150 \mathrm{~s}$. Top row shows global refinement, middle row local prerefinement, and in the last two rows predictorcorrector refinement with $C=0.6$ and $C=0.8$ threshold, respectively. 
Figure 14: Example 3: Performance of the Newton solver. Left: example of the nonlinear residual and elements in the active set converging at the same time for a particular time step. Right: average number of Newton iterations divided into different parts: no crack growth $(t \leq 85 s)$, beginning of crack growth $(86 s \leq t \leq 95 s)$, and crack growth $(t>95 s)$.
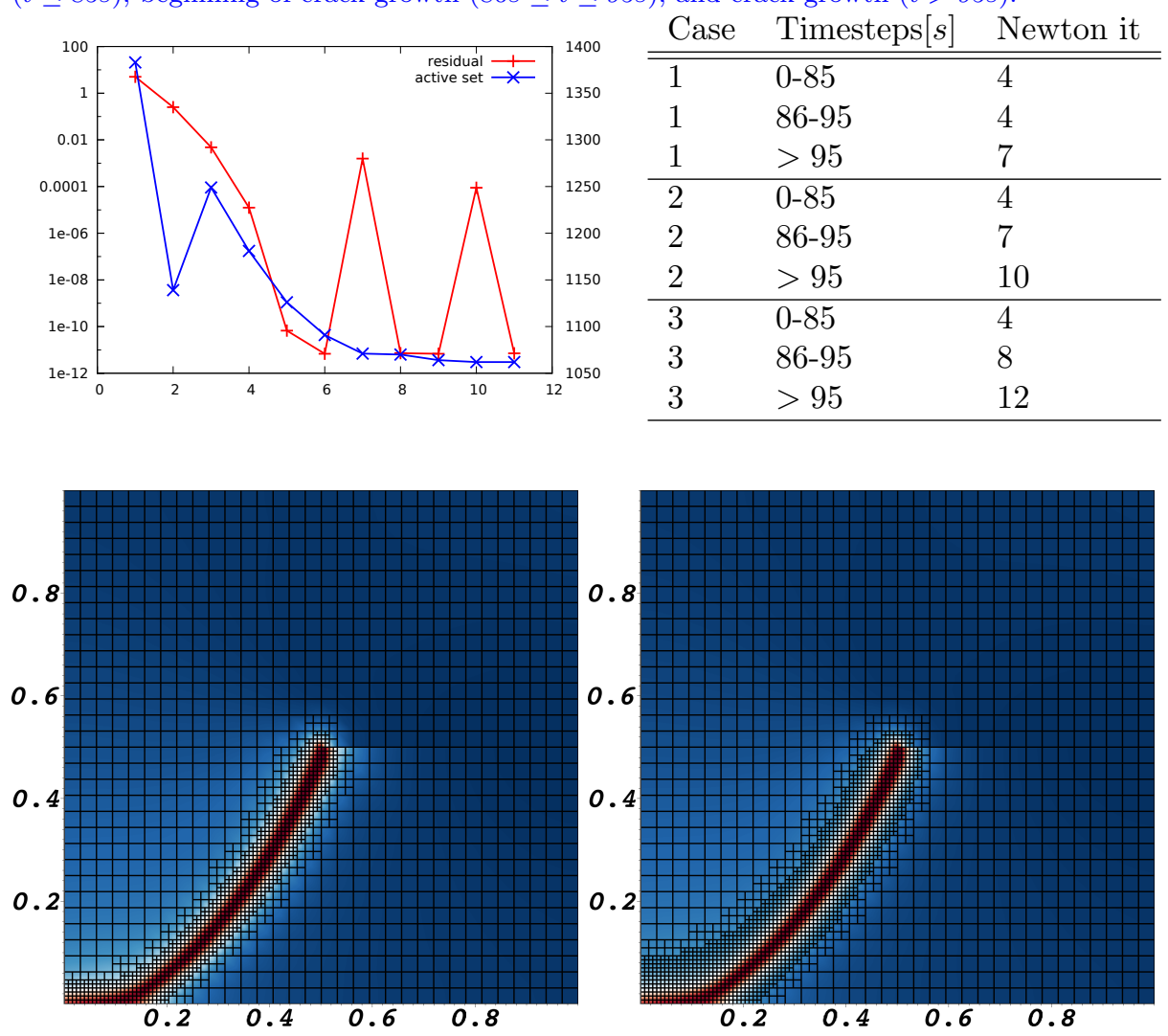

Figure 13: Example 3: Single edge notched shear test. Comparison of the mesh refinement effect using predictor-corrector adaptivity with $C=0.6$ and $C=0.8$ and $4+2$-refinement at the final time step $150 \mathrm{~s}$. The refined region is more concentrated around the crack when using $C=0.6$.

Performance of the combined Newton solver with respect to $h$ and $\varepsilon$ and the active set constant $c$

For the three different cases $1,2,3$ we provide some details on the convergence of the merged Newton solver on the finest mesh level $4+4$. We observe that the performance of the Newton solver slightly depends on the $h-\varepsilon$ choice and becomes inferior for choices that result in more accurate solutions. See Figure 14. In other experiments, we see very little dependency on the choice of the active set parameter $c$, which might be a property of the combined Newton method. 


\section{Computational cost}

We now describe our findings regarding the computational cost using the single edge notched shear test (Example 3). The tests are run on a Intel(R) Core(TM) i5-3320M CPU @ 2.60GHz machine. We use a four-times globally refined mesh as a basic mesh. Here, we compare global refinement (leading to a six times globally refined mesh), prerefined local mesh refinement in which the mesh is refined in the bottom left quadrant of the domain, and our predictorcorrector scheme. The prerefinement has a reasonable refinement area since the crack has a curved path and we do not know where it will grow in this region. The mesh with a growing number of unknowns for the predictor-corrector scheme can be seen in Figure 12. Here, we use the 0.6 and 0.8 as respective thresholds for mesh refinement. In Figure 15, we display the wall clock time and the corresponding evolution of the degrees of freedom for these four test cases. The average number of degrees of freedom is about one half using predictorcorrector adaptivity compared to prerefined local mesh refinement; and about one fourth as compared to global mesh refinement. All refinement techniques show an increase in computing time around time step 95 when the crack starts to grow. At time step 126 the crack reaches its final state as observed in Figure 12 (bottom right). In the test with 0.6 threshold, the mesh was changed 32 times, which means that we had to solve 32 additional systems. In the test with 0.8 threshold, the mesh was changed 42 times. This is reasonable since 0.8 marks more cells in a larger region around the crack. These additional solves however still kept the average computing time much below the locally pre-refined configuration. The computational time, number of steps, and corresponding degrees of freedom are provided in Table 1; and showing the computational savings using predictor-corrector adaptivity.

Table 1: Example 3: Comparison of computational cost for different refinement strategies.

\begin{tabular}{l|r|l|c} 
& Time/s & Number of steps & DoFs: min/avg/max \\
\hline \hline global refinement & 5036 & 151 & 50115 \\
local prerefinement & 1277 & 151 & 19746 \\
predictor/corrector (0.8) & 233 & $151+63$ & $3315 / 4731 / 8286$ \\
predictor/corrector (0.6) & 184 & $151+53$ & $3315 / 4225 / 6666$ \\
\hline
\end{tabular}



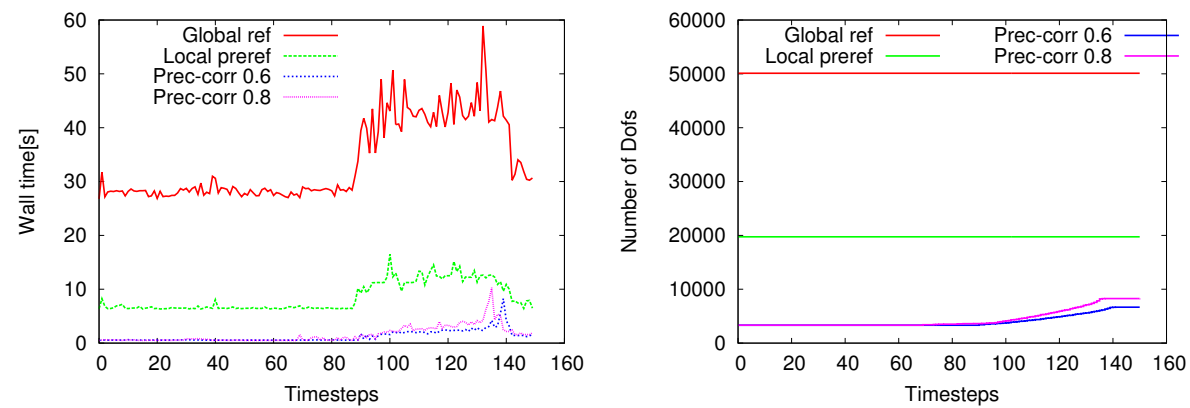

Figure 15: Example 3: Single edge notched shear test on 4+2-refined meshes. Comparison of computational cost in terms of the wall clock time (left) and corresponding evolution of the degrees of freedom. The wall clock time is measured for each time step. Using the predictor-corrector scheme it summarizes all states in which the mesh might be refined and consequently one time step includes possible solution of more than one system solve. The right figure displays that the total number of increases while the crack growths. Even in the final steps, when the crack reaches the boundary (see Figure 12), the number of DoFs is much smaller in the adaptive approach.

\subsection{Symmetric three point bending (Example 4)}

This example is a classical benchmark in mechanical engineering. Related studies have been carried out in [39] and by the same authors with a phase-field method $[4,5]$. Other important results of related settings have been reported in [40]. Here, we have two goals in mind:

- further application of our predictor-corrector mesh adaptivity scheme in order to perform mesh sensitivity studies by disturbing the mesh, while observing the crack path and functional values;

- as in the previously examples studies with respect to $h$ and $\varepsilon$;

The geometric, material properties and loading conditions are the same as used in $[40,39,5]$. The configuration is displayed in Figure 16. 


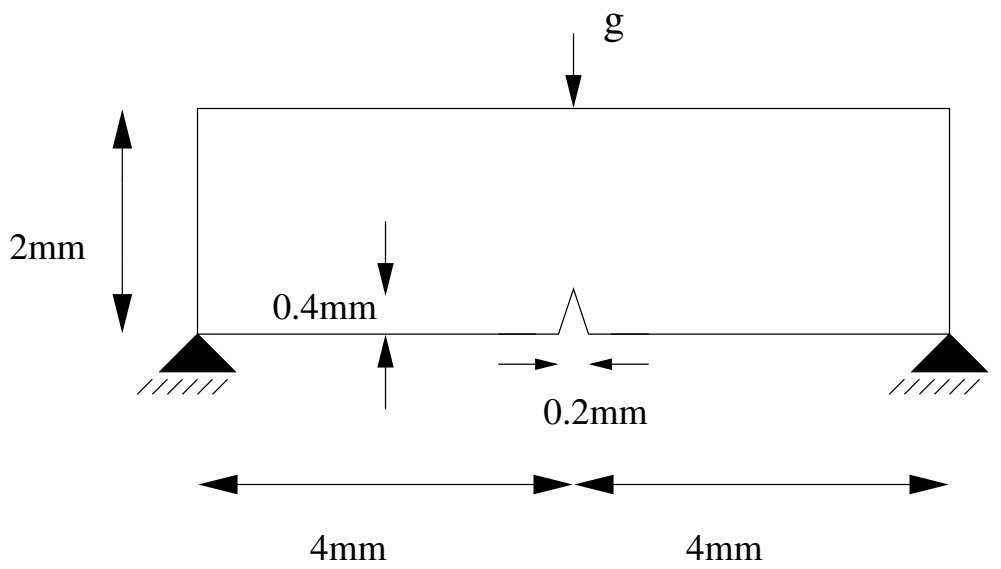

Figure 16: Example 4: Configuration of the symmetric three point bending test. A time dependent Dirichlet force $u_{y}$ acts on $\Gamma_{t o p}$ only in the middle, i.e., $\boldsymbol{g}$ in the point $(4 \mathrm{~mm}, 2 \mathrm{~mm})$ on $\Gamma_{\text {top }}$ (the origin being in the left corner on the bottom). In the two bottom corners, the specimen is fixed only by $\boldsymbol{u}_{y}=0 \mathrm{~mm}$ and traction-free in $x$-direction. To fix rigid body motions, we also clamp one pin in $x$-direction, $u_{x}=0$. All remaining boundary parts are traction-free, i.e., homogeneous Neumann conditions.

The material parameters are $\lambda=12 \mathrm{kN} / \mathrm{mm}^{2}, \mu=8 \mathrm{kN} / \mathrm{mm}^{2}$ and $G_{c}=$ $2.5 \times 10^{-4} \mathrm{kN} / \mathrm{mm}^{2}$. We increase the displacement $\boldsymbol{g}$ in $(4 \mathrm{~mm}, 2 \mathrm{~mm})$ on $\Gamma_{t o p}$ over time, namely we apply again a non-homogeneous Dirichlet:

$$
\boldsymbol{g}=t \overline{\boldsymbol{u}}, \quad \overline{\boldsymbol{u}}=1 \mathrm{~mm} / \mathrm{s}
$$

where $t$ denotes the total time. The time step size is chosen as $10^{-3} s$ in the first 35 steps and afterwards $10^{-5} \mathrm{~s}$ due to brutal and unstable crack growth. The load $F_{y}:=\sigma \cdot n$ is measured along the top boundary.

As previously mentioned, our main goal in this test are mesh sensitivity studies. Here, two initial meshes symm with 312 cells and non-symm with 632 cells differ in their number of initial cells and their symmetry. The non-symm mesh is highly distorted in order to study independence of crack growth with respect to the geometry and mesh. In addition, we finally disturb the initial symmetric mesh symm by $30 \%$ obtaining symm - dist in order to make another comparison with disturbed meshes.

We first observe that the crack path is not affected and the growth and its path is insensitive of the mesh, which is demonstrated in the Figures 19 23. Moreover, we also see that using the different initial, non-symmetric mesh non - symm, the load-displacement curves are of similar order as observed in the Figures 17 and 18. Most importantly, crack growth starts independently at exactly the same time instance $t=0.036 \mathrm{~s}$ in all tests. 

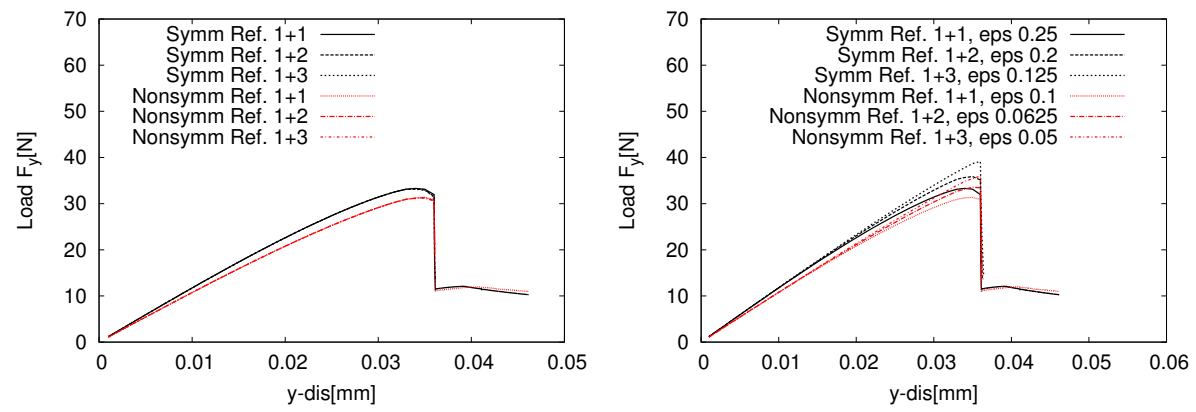

Figure 17: Example 4: symmetric three point bending test - comparison of symm and nonsymm meshes for fixed $\varepsilon=2 h_{\text {min }}^{\text {coarse }}$ and $h$-refinement (left figure). The functional values slightly differ, which is due to the different numbers of cells due to different initially generated meshes. In the right figure, $\varepsilon$ depends on $h$ by $\varepsilon=2 h_{m i n}$. The starting point of crack growth is the same for all parameter choices. However, we refrain from speaking of 'convergence' in the case of $\varepsilon$-refinement (in the right figure) since convergence of the functional curves for $t \leq 0.036 s$ is not yet completely satisfied.
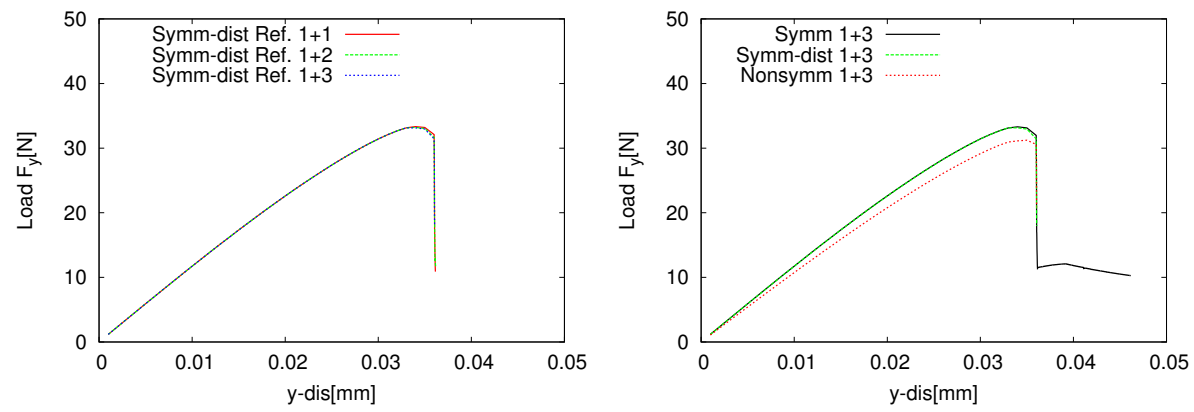

Figure 18: Example 4: symmetric three point bending test. The initial symm mesh is disturbed by $30 \%$ and $h$-convergence is studied with respect to fixed $\varepsilon=2 h_{\text {min }}=0.2$. At right, the three meshes are compared for a specific choice on the finest mesh $1+3$ and fixed $\varepsilon$. Again, the starting point of crack growth is for all meshes the same. The slight difference in the functional curves stems from a different number of mesh cells using the symm or non - symm meshes. This is confirmed by the fact that the second distorted mesh symm - dist (obtained from symm, and consequently the same number of mesh cells) does not show any discrepancy in the functional curves. 


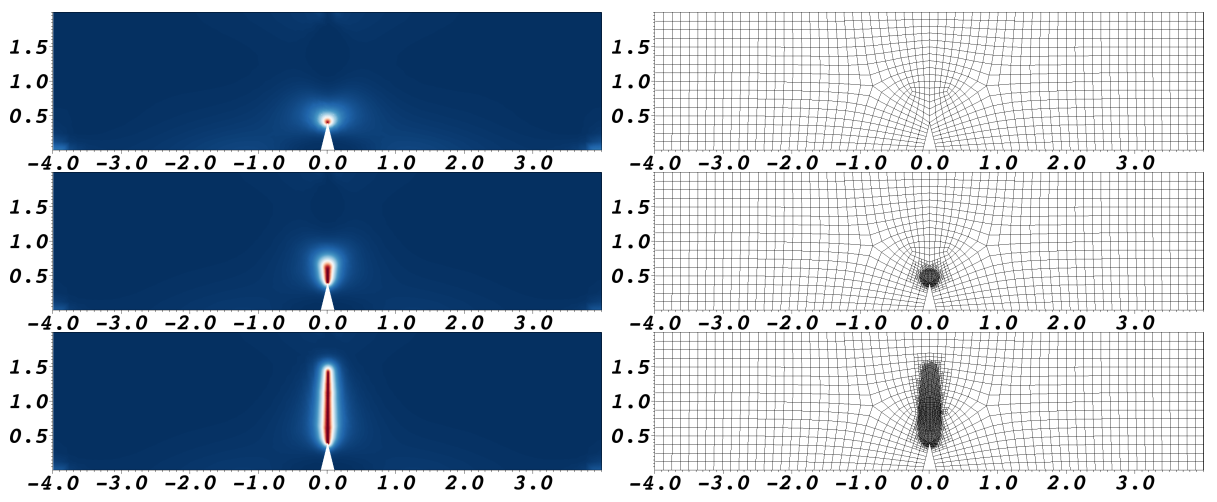

Figure 19: Example 4: Symmetric three point bending test using the symm mesh: snapshots at different times $t=0 s, 0.035 s, 0.03604 s, 0.037 s$.

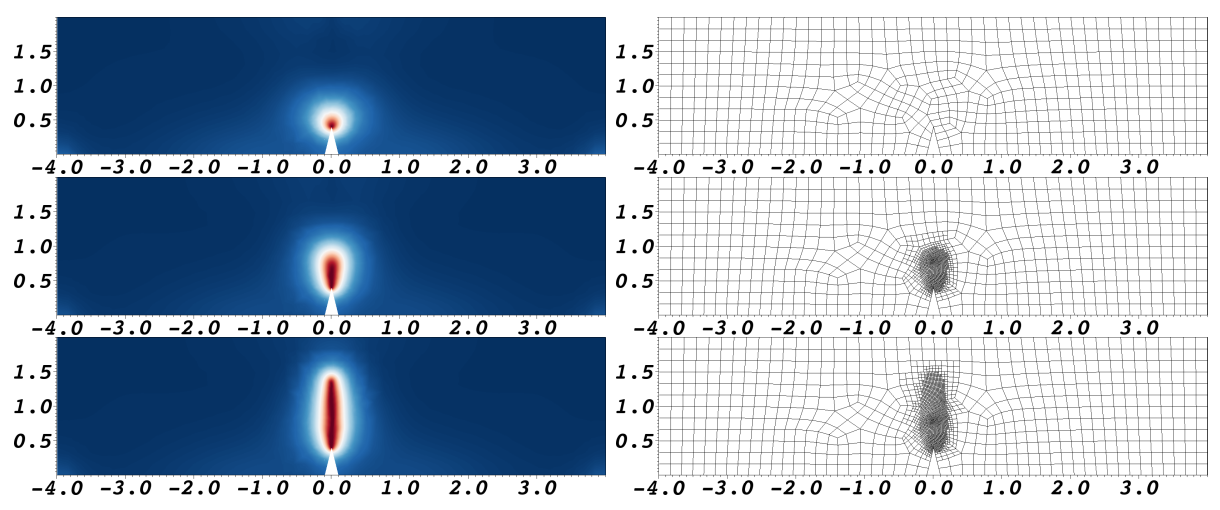

Figure 20: Example 4: Symmetric three point bending test using the non - symm mesh: snapshots at different times $t=0 s, 0.035 s, 0.03604 s, 0.037 s$.

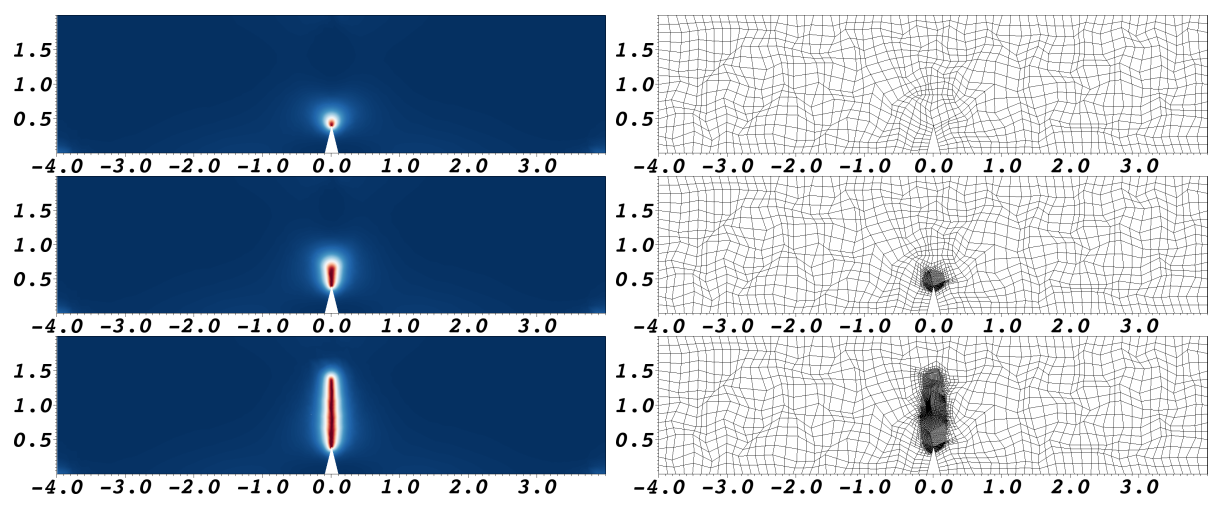

Figure 21: Example 4: Symmetric three point bending test using the symm - dist mesh: snapshots at different times $t=0 s, 0.035 s, 0.03604 s, 0.037 s$. 


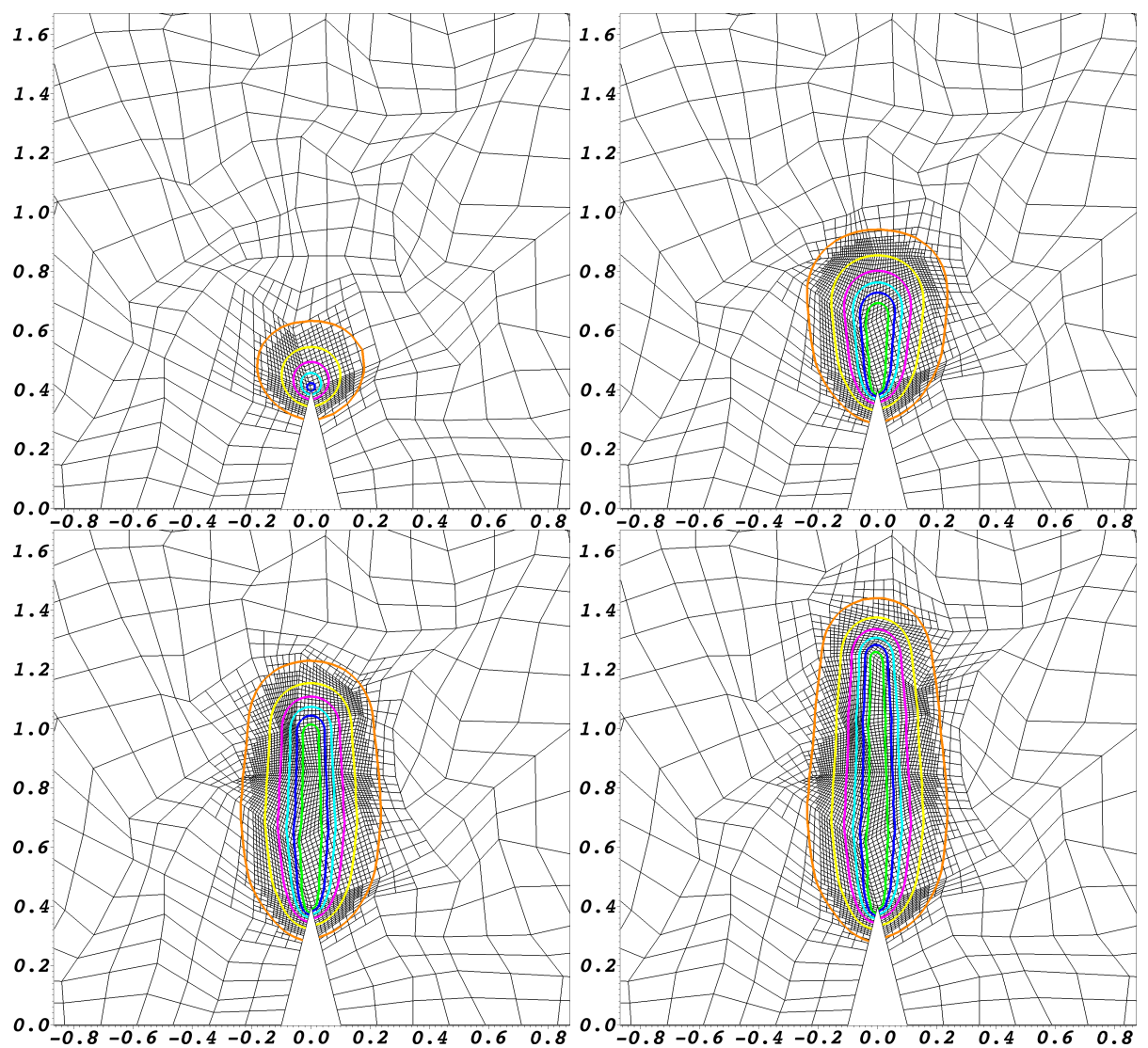

Figure 22: Example 4: Symmetric three point bending test contour plots of the crack surfaces showing their symmetry while the mesh is non-symmetric (using the symm-dist mesh). 


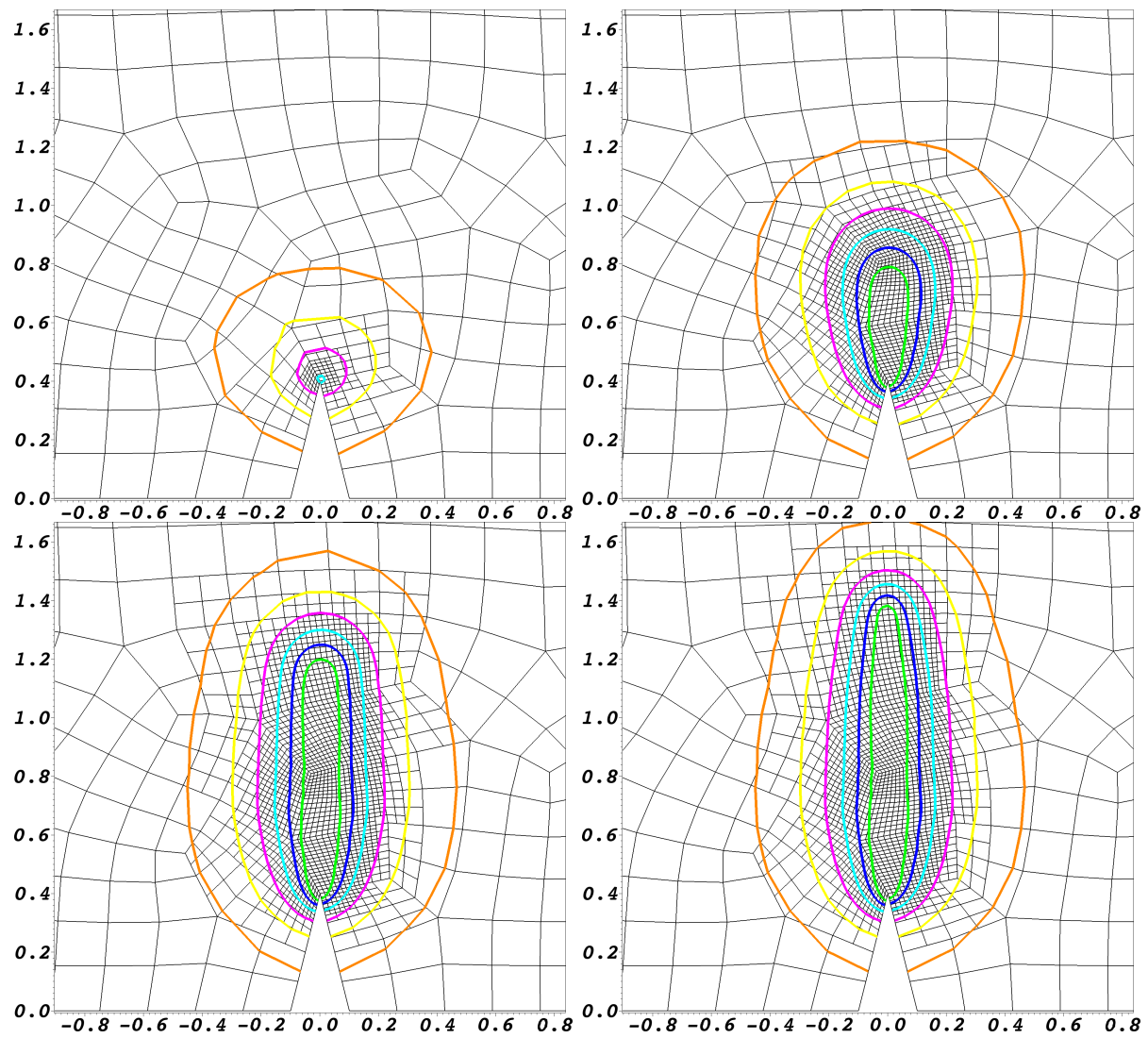

Figure 23: Example 4: Symmetric three point bending test contour plots of the crack surfaces showing their symmetry while the mesh is non-symmetric (using the non - symm mesh).

\subsection{Multiple pressurized cracks (Examples 5 and 6)}

In this example, we employ our predictor-corrector dynamic mesh adaptivity algorithm for propagating pressurized fractures. We also demonstrate that branching and joining in heterogeneous media are automatically captured by the phase-field method. Specifically, we consider two propagating fractures that interact with each other.

We use the same initial domain as in Example 1. In Example 5, two parallel cracks in $\Omega_{C}=[1.6 m, 2.4 m] \times[2.75 m-h, 2.75 m+h]$ and $\Omega_{C}=$ $[1.6 m, 2.4 m] \times[1.25 m-h, 1.25 m+h]$ are prescribed. In Example 6, a vertical crack on $\Omega_{C}=[0.5 m, 1.5 m] \times[3.0 m-h, 3.0 m+h]$ and a horizontal crack on $\Omega_{C}=[2.5 m-h, 2.5 m+h] \times[0.8 m, 1.5 m]$ are initially given. The corresponding meshes are first 4 times globally refined with 4 additional levels of predictor-corrector adaptive refinement. For these additional refinements, the phase-field variable is used with the threshold 0.8. The time step is $k=0.01 s$. 
The regularization parameters are chosen as $\kappa=10^{-10} \mathrm{~m}$ and $\varepsilon=6 h_{\text {min }}^{0.5} \mathrm{~m}$. In this test, the crack propagates due to a pressure that increases linearly in time:

$$
p(t)=t \cdot 10^{3}[\mathrm{~Pa}] .
$$

The material parameters for Example 5 are $E=10^{4} \mathrm{~Pa}$ and $\nu_{s}=0.2$ and for the second heterogeneous Example 6 we choose $E \in\left[1,10^{5}\right] P a$; and $\nu_{s}=0.2$ as before.

Our findings show that cost-efficient complex crack topologies can be computed with our proposed approach. Specifically, we observe nonplanar crack growth with branching and joining of two fractures in Example 6. In Figure 24 the initial and final meshes of Example 5 are displayed. The evolution of the two cracks can be observed in Figure 26 for a sequence of different time snapshots.

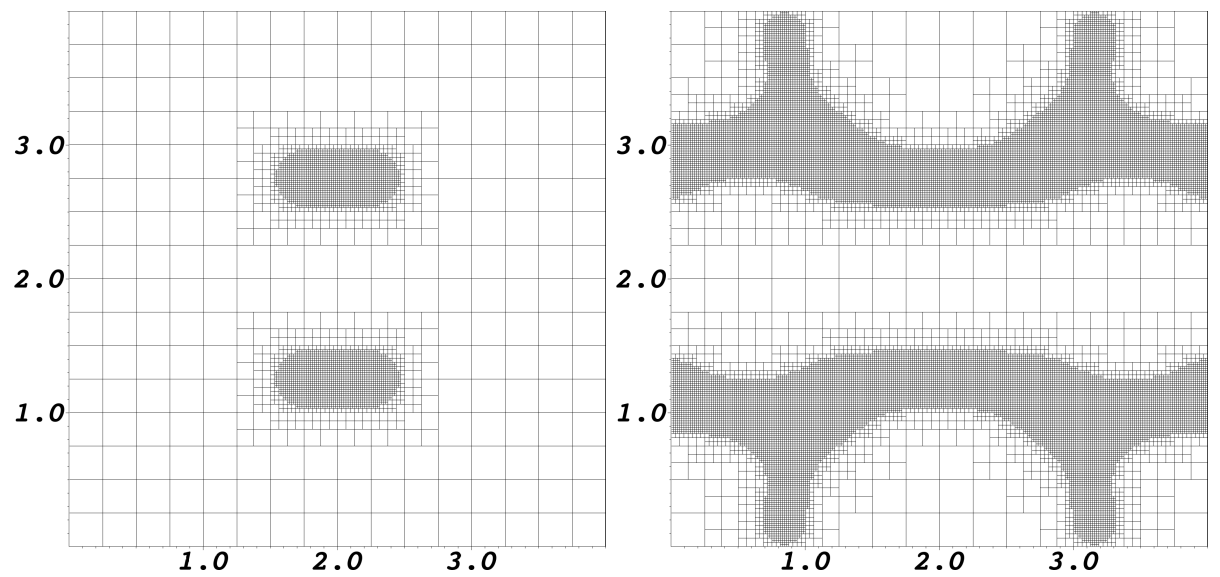

Figure 24: Example 5: Initial and final meshes at $T=0 \mathrm{~s}$ and $T=30 \mathrm{~s}$. 

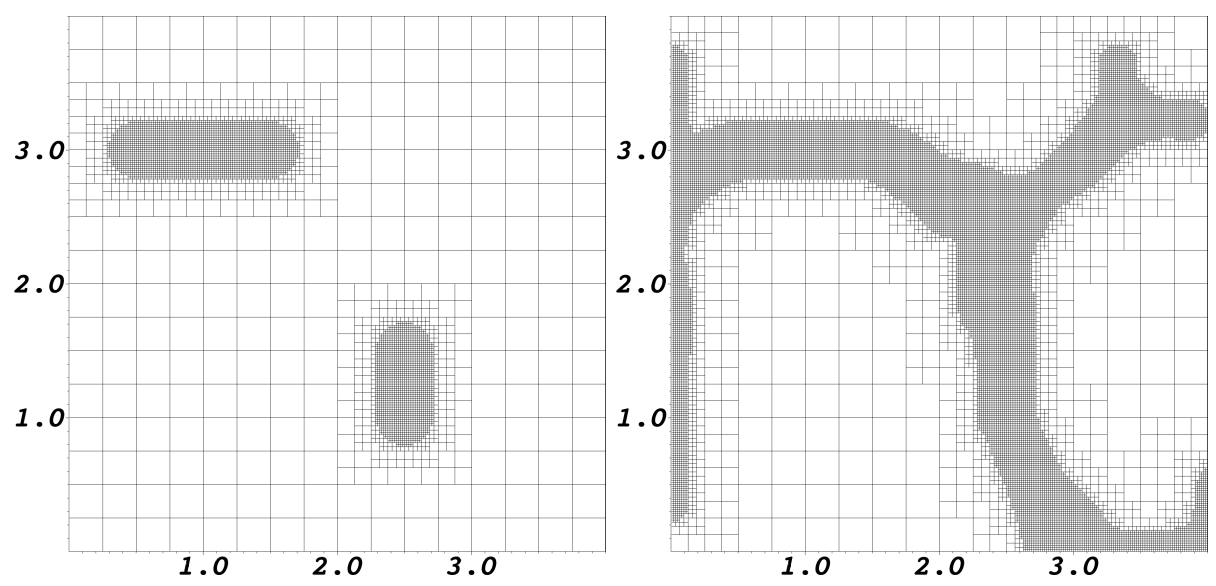

Figure 25: Example 6: Initial and final meshes at $T=0 \mathrm{~s}$ and $T=50 \mathrm{~s}$. 

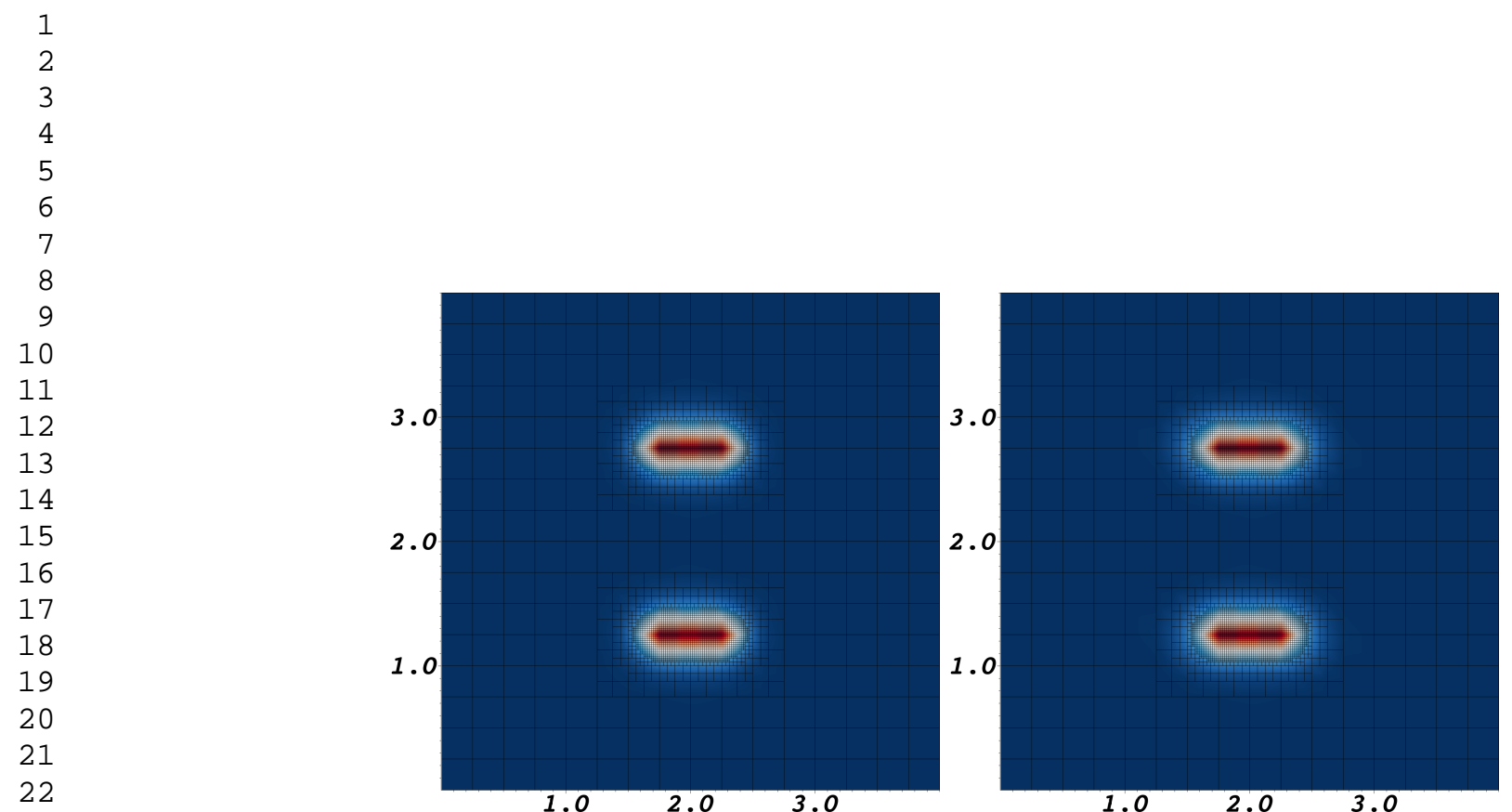

23

24

25

26

27

28

29

30

31

32

33

34

35

36

37

38

39

40

41

42

43

44

45

46

47

48

49

50

51

52

53

54

55

56

57

58

59

60

61

62

63 


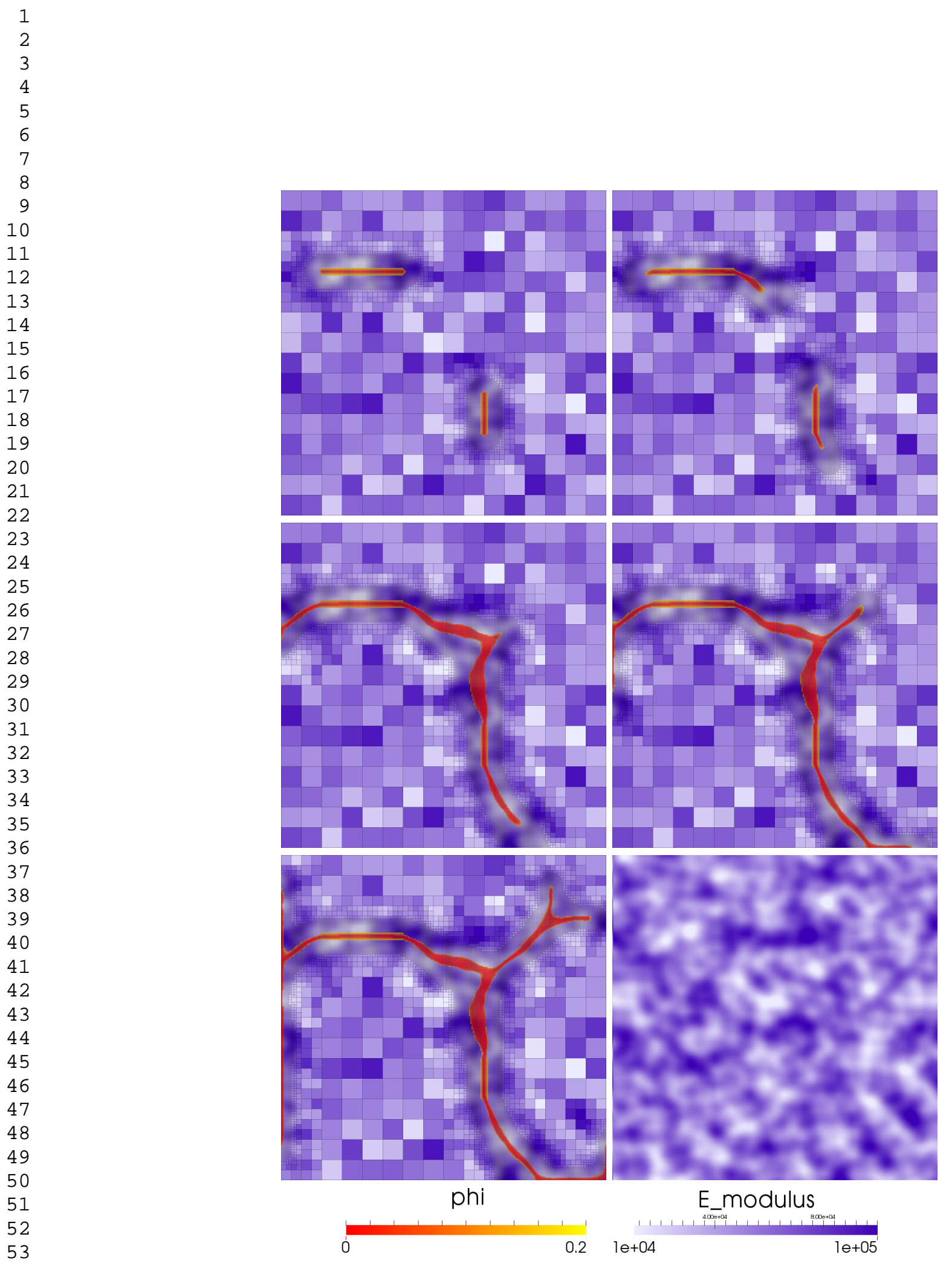

Figure 27: Example 6: Two propagating pressurized cracks at $T=0 s, 30 s, 35 s, 40 s, 50 s$ and the E-modulus used in the computation. Here, both fractures grow nonplanar, then they join, and finally the crack branches in the top right corner. 
The results of Example 6 are shown in the Figures 25 and 27. Here, the cracks first grow nonplanarly, join, and then grow towards the boundary before the crack branches again, which highlights the capabilities of the phase-field method. We note that the physical behavior is dependent on the boundary conditions and might change for other configurations. Finally, the bulk and crack energies are provided in Figure 28. As before, the crack energy increases when the cracks starts growing. In contrast to the previous examples, the bulk energy is still increasing since we keep increasing the pressure.
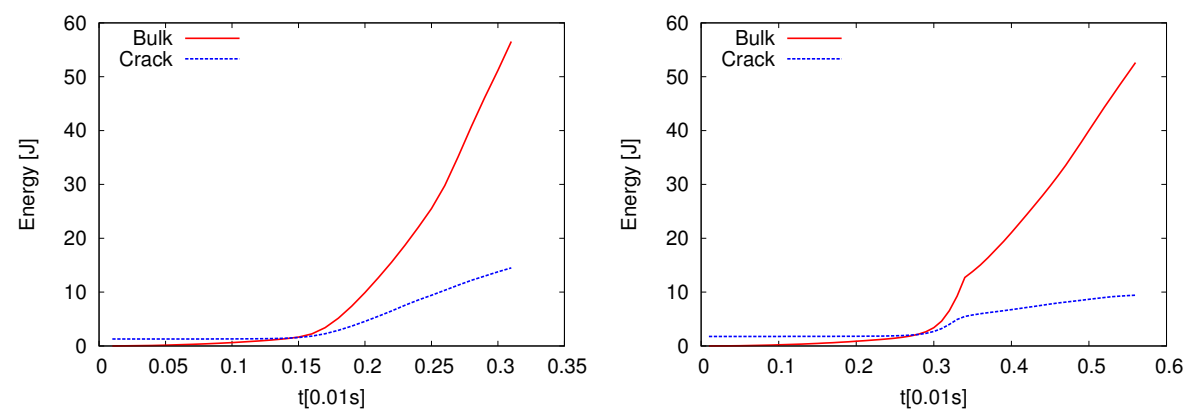

Figure 28: Examples 5 and 6: Comparison of bulk and crack energies.

\section{Conclusions}

In this work, we formulated a primal-dual active set method with predictorcorrector mesh adaptivity for solving fracture problems with a phase-field method. Our results demonstrate that the approach is computationally robust, efficient, and accurate. We include investigations between the regularization parameter $\varepsilon$, the mesh size parameter $h$, and the time step size $\delta t$ to study both spatial and temporal convergence. Moreover, we investigated computational cost and time, and the performance of Newton's method with respect to these different parameters. In addition, studies showing insensitivity of crack growth with respect to the mesh structure have been performed. All studies have been computed using acknowledged benchmark tests such as Sneddon's test for pressurized fractures as well as shear tests and three point bending tests in elasticity.

\section{Acknowledgments}

The first author thanks the Center for Subsurface Modeling at ICES, UT Austin, for hosting his research stay in June 2014. Additionally, the first author is supported in part through the Computational Infrastructure in Geodynamics initiative (CIG), through the National Science Foundation under Award No. EAR-0949446 and The University of California - Davis. The third author is grateful to the Alexander von Humboldt foundation and an ICES postdoctoral fellowship for supporting his work at ICES. This research was funded by 
ConocoPhillips grant UTA10-000444, DOE grant ER25617, Saudi Aramco grant UTA11-000320 and Statoil grant UTA13-000884. The authors would like to express their sincere thanks for the funding. Finally, we would like to thank the reviewers for their excellent comments that allowed us to improve the paper.

\section{Appendix 1: Strain split into tension and compression}

The decomposition of the strain energy (i.e., of the resulting stress tensors) is based on a spectral decomposition into the principal components of the strain tensor into positive and negative components, which are then used to build the stress tensor. We recapitulate: A spectral decomposition of the strain tensor is given by:

$$
e(\boldsymbol{u})=M \Lambda M^{T}
$$

where $M=\left(v_{1}, v_{2}\right)$ is build by the orthonormal eigenvectors of $e(\boldsymbol{u})$ and $\Lambda=$ $\operatorname{diag}\left(\lambda_{1}, \lambda_{2}\right)$, where $\lambda_{i}:=\lambda_{i}(\boldsymbol{u})$, denote the principal strains and $v_{i}:=v_{i}(\boldsymbol{u})$ the corresponding eigenvectors. Define

$$
e^{+}(\boldsymbol{u})=M \Lambda^{+} M^{T}, \quad \text { and } \quad e^{-}(\boldsymbol{u})=M \Lambda^{-} M^{T}
$$

with

$$
\Lambda^{+}=\operatorname{diag}\left(\left\langle\lambda_{1}\right\rangle,\left\langle\lambda_{2}\right\rangle\right)
$$

with $\langle\lambda\rangle=\max (\lambda, 0)$. Moreover, $\Lambda^{-}=\Lambda-\Lambda^{-}$.

Working in two dimensions, allows for the following explicit calculation of the eigenvalues and eigenvectors:

$$
\begin{aligned}
& \lambda_{1}(\boldsymbol{u})=\frac{\operatorname{tr}(e)}{2}+\sqrt{\frac{\operatorname{tr}(e)^{2}}{4}-\operatorname{det}(e),} \\
& \lambda_{2}(\boldsymbol{u})=\frac{\operatorname{tr}(e)}{2}-\sqrt{\frac{\operatorname{tr}(e)^{2}}{4}-\operatorname{det}(e)} .
\end{aligned}
$$

For a symmetric tensor as it is the case for $e$, the eigenvectors are already orthogonal. It remains to orthonormalize them. We obtain:

$$
\begin{aligned}
& v_{1}(u)=\frac{1}{\sqrt{1+\left(\frac{\lambda_{1}-e_{11}}{e_{12}}\right)^{2}}}\left(\begin{array}{c}
1 \\
\frac{\lambda_{1}-e_{11}}{e_{12}}
\end{array}\right), \\
& v_{2}(u)=\frac{1}{\sqrt{1+\left(\frac{\lambda_{2}-e_{11}}{e_{12}}\right)^{2}}}\left(\begin{array}{c}
1 \\
\frac{\lambda_{2}-e_{11}}{e_{12}}
\end{array}\right) .
\end{aligned}
$$

Remark 6.1 (Strain energy decomposition in 3d). Borden et al. [41] provide some useful hints on the calculation of the strain energy decomposition in three dimensions. 


\section{Appendix 2: Derivation of the derivatives}

We would like to identify optimal convergence properties of our Newton method and derive exact expressions for the directional derivatives of

$\sigma^{+}(\delta \boldsymbol{u}), \sigma^{-}(\delta \boldsymbol{u}), e^{+}(\delta \boldsymbol{u}), \Lambda^{+}(\delta \boldsymbol{u})$ and $P(\delta \boldsymbol{u})$. The key variables to build these derivatives are the eigenvalues and their eigenvectors:

$$
\begin{aligned}
\lambda_{1}^{\prime}(\boldsymbol{u})(\delta \boldsymbol{u})= & \frac{\operatorname{tr}(e(\delta \boldsymbol{u}))}{2}+\frac{1}{2 \sqrt{\frac{\operatorname{tr}(e)^{2}}{4}-\operatorname{det}(e)}}\left(e_{12}(\delta \boldsymbol{u}) e_{21}+e_{12} e_{21}(\delta \boldsymbol{u})+\right. \\
& \left.\frac{\left(e_{11}-e_{22}\right)\left(e_{11}(\delta \boldsymbol{u})-e_{22}(\delta \boldsymbol{u})\right)}{2}\right), \\
\lambda_{2}^{\prime}(\boldsymbol{u})(\delta \boldsymbol{u})= & \frac{\operatorname{tr}(e(\delta \boldsymbol{u}))}{2}-\frac{1}{2 \sqrt{\frac{\operatorname{tr}(e)^{2}}{4}-\operatorname{det}(e)}}\left(e_{12}(\delta \boldsymbol{u}) e_{21}+e_{12} e_{21}(\delta \boldsymbol{u})+\right. \\
& \left.\frac{\left(e_{11}-e_{22}\right)\left(e_{11}(\delta \boldsymbol{u})-e_{22}(\delta \boldsymbol{u})\right)}{2}\right),
\end{aligned}
$$

For the eigenvectors, we differentiate (25) using the product rule, chain rule and quotient rule. Define:

$$
\left\|v_{1}\right\|:=\sqrt{1+\left(\frac{\lambda_{1}-e_{11}}{e_{12}}\right)^{2}}
$$

and

$$
\left\|v_{2}\right\|:=\sqrt{1+\left(\frac{\lambda_{2}-e_{11}}{e_{12}}\right)^{2}},
$$

Then, using the product rule:

$$
\begin{aligned}
v_{1}^{\prime}(\boldsymbol{u})(\delta \boldsymbol{u}) & =\left(\frac{1}{\left\|v_{1}\right\|}\left(\begin{array}{c}
1 \\
\frac{\lambda_{1}-e_{11}}{e_{12}}
\end{array}\right)\right)^{\prime}(\delta \boldsymbol{u}) \\
& =\left(\frac{1}{\left\|v_{1}\right\|}\right)^{\prime}(\delta \boldsymbol{u})\left(\begin{array}{c}
1 \\
\frac{\lambda_{1}-e_{11}}{e_{12}}
\end{array}\right)+\frac{1}{\left\|v_{1}\right\|}\left(\left(\begin{array}{c}
1 \\
\frac{\lambda_{1}-e_{11}}{e_{12}}
\end{array}\right)\right)^{\prime}(\delta \boldsymbol{u}),
\end{aligned}
$$

where

$$
\begin{aligned}
\left(\frac{1}{\left\|v_{1}\right\|}\right)^{\prime}(\delta \boldsymbol{u})= & \left(-\frac{1}{1+\left(\frac{\lambda_{1}-e_{11}}{e_{12}}\right)^{2}} \frac{1}{2 \sqrt{\left(\frac{\lambda_{1}-e_{11}}{e_{12}}\right)^{2}}}\right. \\
& \left.2 \frac{\lambda_{1}-e_{11}}{e_{12}} \frac{\left(\lambda_{1}(\delta \boldsymbol{u})-e_{11}(\delta \boldsymbol{u})\right) e_{12}-\left(\lambda_{1}-e_{11}\right) e_{12}(\delta \boldsymbol{u})}{e_{12}^{2}}\right)
\end{aligned}
$$


Analogously for $\left(\frac{1}{\left\|v_{2}\right\|}\right)^{\prime}(\delta \boldsymbol{u})$. It remains to differentiate the second part $\left(\left(\begin{array}{c}1 \\ \frac{\lambda_{1}-e_{11}}{e_{12}}\end{array}\right)\right)^{\prime}(\delta \boldsymbol{u})$. Here, we obtain with the quotient rule:

$$
\left(\left(\begin{array}{c}
1 \\
\frac{\lambda_{1}-e_{11}}{e_{12}}
\end{array}\right)\right)^{\prime}(\delta \boldsymbol{u})=\left(\begin{array}{c}
0 \\
\frac{\left(\lambda_{1}(\delta \boldsymbol{u})-e_{11}(\delta \boldsymbol{u})\right) e_{12}-\left(\lambda_{1}-e_{11}\right) e_{12}(\delta \boldsymbol{u})}{e_{12}^{2}}
\end{array}\right) .
$$

Now, we insert these derivatives into their defining tenors.

Finally we emphasize that the positive parts $\langle\cdot\rangle, e^{+}$and $\Lambda^{+}$are taken with respect to the right hand side. Specifically, for the entries in the Jacobian, we check if the corresponding right hand side value is non-negative. This ensures that each non-negative component of the right hand side has a corresponding (possibly negative) matrix entry.

\section{References}

[1] G. Francfort, J.-J. Marigo, Revisiting brittle fracture as an energy minimization problem, J. Mech. Phys. Solids 46 (1998) 1319-1342.

[2] B. Bourdin, G. Francfort, J.-J. Marigo, Numerical experiments in revisited brittle fracture, J. Mech. Phys. Solids 48 (2000) 797-826.

[3] B. Bourdin, G. Francfort, J.-J. Marigo, The variational approach to fracture, J. Elasticity 91 (2008) 1-148.

[4] C. Miehe, F. Welschinger, M. Hofacker, Thermodynamically consistent phase-field models of fracture: variational principles and multi-field fe implementations, International Journal of Numerical Methods in Engineering 83 (2010) 1273-1311.

[5] C. Miehe, M. Hofacker, F. Welschinger, A phase field model for rateindependent crack propagation: Robust algorithmic implementation based on operator splits, Comput. Meth. Appl. Mech. Engrg. 199 (2010) 27652778 .

[6] M. J. Borden, C. V. Verhoosel, M. A. Scott, T. J. R. Hughes, C. M. Landis, A phase-field description of dynamic brittle fracture, Comput. Meth. Appl. Mech. Engrg. 217 (2012) 77-95.

[7] M. Artina, M. Fornasier, S. Micheletti, S. Perotto, Anisotropic mesh adaptation for crack detection in brittle materials, 2014. TUM Report.

[8] S. Burke, C. Ortner, E. Süli, An adaptive finite element approximation of a generalized Ambrosio-Tortorelli functional, M3AS 23 (2013) 1663-1697.

[9] N. Moes, J. Dolbow, T. Belytschko, A finite element method for crack growth without remeshing, Int. J. Numer. Methods Engrg. 46 (1999) 131150. 
[10] I. Babuska, U. Banerjee, Stable generalized finite element method (sgfem), Comput. Methods Appl. Mech. Engrg. 201-204 (2012) 91-111.

[11] N. Kikuchi, J. Oden, Contact problems in elasticity, Studies in Applied Mathematics, Society for Industrial and Applied Mathematics (SIAM), Philadelphia, PA, 1988.

[12] A. Mikelić, M. Wheeler, T. Wick, A phase-field approach to the fluid filled fracture surrounded by a poroelastic medium, 2013. ICES Report 13-15.

[13] A. Mikelić, M. Wheeler, T. Wick, A quasi-static phase-field approach to the fluid filled fracture, 2013. ICES Report 13-22, submitted for publication.

[14] A. Mikelić, M. Wheeler, T. Wick, Phase-field modeling of pressurized fractures in a poroelastic medium, 2014. ICES-Preprint 14-18, submitted for publication.

[15] M. Hintermüller, K. Ito, K. Kunisch, The primal-dual active set strategy as a semismooth newton method, SIAM Journal on Optimization 13 (2002) 865-888.

[16] B. Wohlmuth, Variationally consistent discretization schemes and numerical algorithms for contact problems, Acta Numerica 20 (2011) 569-734.

[17] J. Nocedal, S. J. Wright, Numerical optimization, Springer Ser. Oper. Res. Financial Engrg., 2006.

[18] A. Settari, D. A. Walters, Advances in coupled geomechanical and reservoir modeling with applications to reservoir compaction, SPE Journal 6 (2001) 334-342.

[19] A. Mikelić, M. F. Wheeler, Convergence of iterative coupling for coupled flow and geomechanics, Comput Geosci 17 (2012) 455-462.

[20] A. Mikelić, M. Wheeler, T. Wick, A phase-field method for propagating fluid-filled fractures coupled to a surrounding porous medium, 2014. ICES Report 14-08, submitted for publication.

[21] T. Wick, G. Singh, M. Wheeler, Pressurized fracture propagation using a phase-field approach coupled to a reservoir simulator, 2013. SPE 168597MS, SPE Proc.

[22] T. Wick, G. Singh, M. Wheeler, Fluid-filled fracture propagation using a phase-field approach and coupling to a reservoir simulator, 2014. ICES report 14-20.

[23] K. Pham, H. Amor, J.-J. Marigo, C. Maurini, Gradient Damage Models and Their Use to Approximate Brittle Fracture, Int. J. of Damage Mech. (2011) $1-36$. 
[24] L. Ambrosio, V. Tortorelli, Approximation of functionals depending on jumps by elliptic functionals via $\gamma$-convergence, Comm. Pure Appl. Math. 43 (1990) 999-1036.

[25] L. Ambrosio, V. Tortorelli, On the approximation of free discontinuity problems, Boll. Un. Mat. Ital. B 6 (1992) 105-123.

[26] A. Braides, Approximation of Free-Discontinuity Problems, Lecture Notes in Mathematics No. 1694, Springer Verlag, Berlin, 1998.

[27] B. Bourdin, Numerical implementation of the variational formulation for quasi-static brittle fracture, Interfaces and free boundaries 9 (2007) 411430.

[28] S. Burke, C. Ortner, E. Süli, An adaptive finite element approximation of a variational model of brittle fracture, SIAM J. Numer. Anal. 48 (2010) 980-1012.

[29] N. Gould, D. Orban, P. Toint, Numerical methods for large-scale nonlinear optimization, Acta Numerica 14 (2005) 299-361.

[30] M. A. Heroux, R. A. Bartlett, V. E. Howle, R. J. Hoekstra, J. J. Hu, T. G. Kolda, R. B. Lehoucq, K. R. Long, R. P. Pawlowski, E. T. Phipps, A. G. Salinger, H. K. Thornquist, R. S. Tuminaro, J. M. Willenbring, A. Williams, K. S. Stanley, An overview of the trilinos project, ACM Trans. Math. Softw. 31 (2005) 397-423.

[31] M. Negri, The anisotropy introduced by the mesh in the finite element approximation of the Mumford-Shah functional, Numer. Func. Anal. opt. 20 (1999) 957-982.

[32] I. N. Sneddon, M. Lowengrub, Crack problems in the classical theory of elasticity, SIAM series in Applied Mathematics, John Wiley and Sons, Philadelphia, 1969.

[33] W. Bangerth, T. Heister, L. Heltai, G. Kanschat, M. Kronbichler, M. Maier, B. Turcksin, T. D. Young, The deal .ii library, version 8.1, arXiv preprint http://arxiv.org/abs/1312.2266v4 (2013).

[34] W. Bangerth, T. Heister, G. Kanschat, deal. II Differential Equations Analysis Library, Technical Reference, 2013. http://www.dealii.org.

[35] T. Wick, Solving monolithic fluid-structure interaction problems in arbitrary Lagrangian Eulerian coordinates with the deal.ii library, Archive of Numerical Software 1 (2013) 1-19.

[36] J. Frohne, T. Heister, W. Bangerth, Efficient numerical methods for the large-scale parallel solution of elastoplastic contact problems, 2013. Preprint. 
[37] I. N. Sneddon, The distribution of stress in the neighbourhood of a crack in an elastic solid, Proc. R Soc London A 187 (1946) 229-260.

[38] M. Wheeler, T. Wick, W. Wollner, An augmented-Lagangrian method for the phase-field approach for pressurized fractures, Comp. Meth. Appl. Mech. Engrg. 271 (2014) 69-85.

[39] C. Miehe, E. Gürses, A robust algorithm for configurational-force-driven brittle crack propagation with r-adaptive mesh alignment, International Journal for Numerical Methods in Engineering 72 (2007) 127-155.

[40] T. Bittencourt, P. Wawrzynek, A. Ingraffea, J. Sousa, Quasi-automatic simulation of crack propagation for $2 \mathrm{~d}\{\mathrm{LEFM}\}$ problems, Engineering Fracture Mechanics 55 (1996) 321 - 334 .

[41] M. J. Borden, T. J. R. Hughes, C. M. Landis, C. V. Verhoosel, A higherorder phase-field model for brittle fracture: Formulation and analysis within the isogeometric analysis framework, Technical Report ICES Report 13-20, The University of Texas at Austin, 2013. 


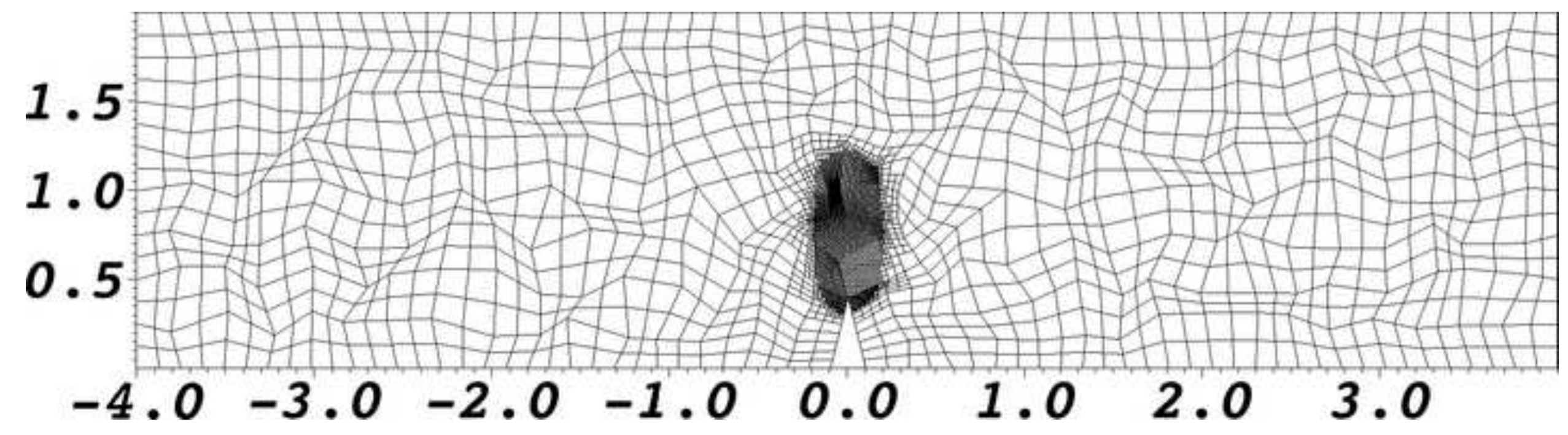

